\title{
Shape Design in Aorto-Coronaric Bypass Anastomoses using Perturbation Theory
}

\author{
Valery Agoshkov ${ }^{1 *}$ Alfio Quarteroni ${ }^{2,3}$, Gianluigi Rozza ${ }^{2}$ \\ ${ }^{1}$ Institute of Numerical Mathematics, \\ Russian Academy of Sciences, \\ 119991 GSP-1 Moscow, Russia. \\ ${ }^{2}$ Chair of Modelling and Scientific Computing (CMCS), \\ Institute of Analysis and Scientific Computing (IACS), \\ École Polytechnique Fédérale de Lausanne, \\ CH-1015, Lausanne, Switzerland. \\ ${ }^{3}$ MOX, Dipartimento di Matematica "Francesco Brioschi", \\ Politecnico di Milano, \\ 20133 Milano, Italy.
}

\section{TECHNICAL REPORT}

27 November 2003

\section{Summary}

In this paper we present a new approach in the study of Aorto-Coronaric bypass anastomoses configurations based on small perturbation theory. The theory of optimal control based on adjoint formulation is applied in order to optimize the shape of the zone of the incoming branch of the bypass (the toe) into the coronary (see Figure (1)). The aim is to provide design indications in the perspective of future development for prosthetic bypasses.

Key words: Optimal Control, Flow Control, Shape Optimization, Adjoint Problem, Small Perturbation Theory, Finite Elements, Generalized Stokes Equations, Haemodynamics, Aorto-Coronaric Bypass Anastomoses, Design of Improved Medical Devices.

\section{Introduction}

We consider the application of the optimal control approaches to investigate the problem of shape optimization of aorto-coronaric bypass anastomoses ([17]).

\footnotetext{
*This work has been prepared when the first author spent a research period at Bernoulli Center of the Swiss Federal Institute of Technology Lausanne in the framework of the "special semester on the Mathematical Modelling of the Cardiovascular System"
} 
We analyze the "first corrections" which are derived by applying a perturbation method to the initial problem in a domain $\Omega \subset \mathbb{R}^{2}$ whose boundary $\partial \Omega$ is parameterized by a suitable function $f$. Then we propose numerical methods for its solutions.

The surgical realization of a bypass to overcome a critically stenosed artery is a very common practice in everyday cardiovascular clinic.

Improvement in the understanding of the genesis of coronary diseases is very important as it allows to reduce surgical and post-surgical failures. It may also suggest new means in bypass surgical procedures with less invasive methods and to devise new shape in bypass configuration ([16].

Generally speaking, mathematical modelling and numerical simulation can allow better understanding of phenomena involved in vascular diseases ([19], [18] and $[6])$.

When a coronary artery is affected by a stenosis, the heart muscle can't be properly oxygenated through blood. Aorto-coronaric anastomosis restores the oxygen amount through a bypass surgery downstream an occlusion.

At present, different kind and shape for aorto-coronaric bypass anastomoses are available and consequently different surgery procedures are used to set up a bypass.

A bypass can be made up either by organic material (e.g. the saphena vein taken from patient's legs or the mammary artery) or by prosthetic material. The current saphenous bypass solution requires the extraction of saphena vein with possible complications. In this respect, prosthetic bypasses are less invasive. They may feature very different shape for bypass anastomoses, such as, e.g., cuffed arteriovenous access grafts. Different cuffed models are used such as Taylor Patch [2] and Miller Cuff Bypass, [4], but also standard end-to-side anastomoses at different graft angle ([3]) or other shaped carbon-fiber prostheses. In the cardiovascular system altered flow conditions such as separation, flow reversal, low and oscillatory shear stress areas and abnormal pulse pattern are all recognized as potentially important factors in the development of arterial diseases ([13] and [15]). For all these different aspects the design of artificial arterial bypass is a very complex problem. Carbon fiber and Collagen cuffed grafts instead of natural saphenous vein can be used for studying new shape design without needing "in loco" reconstruction. In this framework, Optimal Control by perturbation theory provide a new approach to the problem, with the goal of improving arterial bypass graft on the basis of a better understanding of fluid dynamics aspects involved in the bypass studying.

\section{Notation and Problem Statement}

In the sequel we use the following notations: $\Omega$ is a bounded domain of $\mathbb{R}^{2}$, $\Gamma \equiv \partial \Omega$ is the boundary of $\Omega, \bar{\Omega}=\Omega \cup \partial \Omega, \underline{x} \equiv(x, y)$ is a point of $\bar{\Omega}, \underline{v} \equiv(u, v)$. We recall the following symbols:

$$
\begin{gathered}
\phi=\phi(\underline{x})=\phi(x, y), \nabla \phi=\left(\frac{\partial \phi}{\partial x}, \frac{\partial \phi}{\partial y}\right), \\
\nabla \cdot \underline{v} \equiv \operatorname{div}(\underline{v}) \equiv \mathcal{D}(\underline{v})=\frac{\partial u}{\partial x}+\frac{\partial v}{\partial y},
\end{gathered}
$$




$$
\begin{gathered}
\nabla \times \underline{v} \equiv \operatorname{rot}(\underline{v})=\frac{\partial v}{\partial x}-\frac{\partial u}{\partial y}, \\
\underline{\operatorname{rot}}(\phi)=\left(\frac{\partial \phi}{\partial y},-\frac{\partial \phi}{\partial x}\right) .
\end{gathered}
$$

We remind that:

$$
\underline{\operatorname{rot}}(\nabla \times \underline{v})=-\Delta \underline{v}+\nabla(\nabla \cdot \underline{v}), \Delta \phi=\nabla \cdot(\nabla \phi) .
$$

Consider an idealized, two-dimensional bypass bridge configuration in Fig.(1) and the domain on Fig.(2), where the dotted line represents the geometry of the complete anastomosis $\left(\Gamma_{w_{2}}\right.$ is the section of the original artery, $\Gamma_{i n}$ is the new anastomosis inflow after bypass surgery, $\Gamma_{\text {out }}$ is the anastomosis outflow).

We consider the following boundary value problem for the Stokes equations:

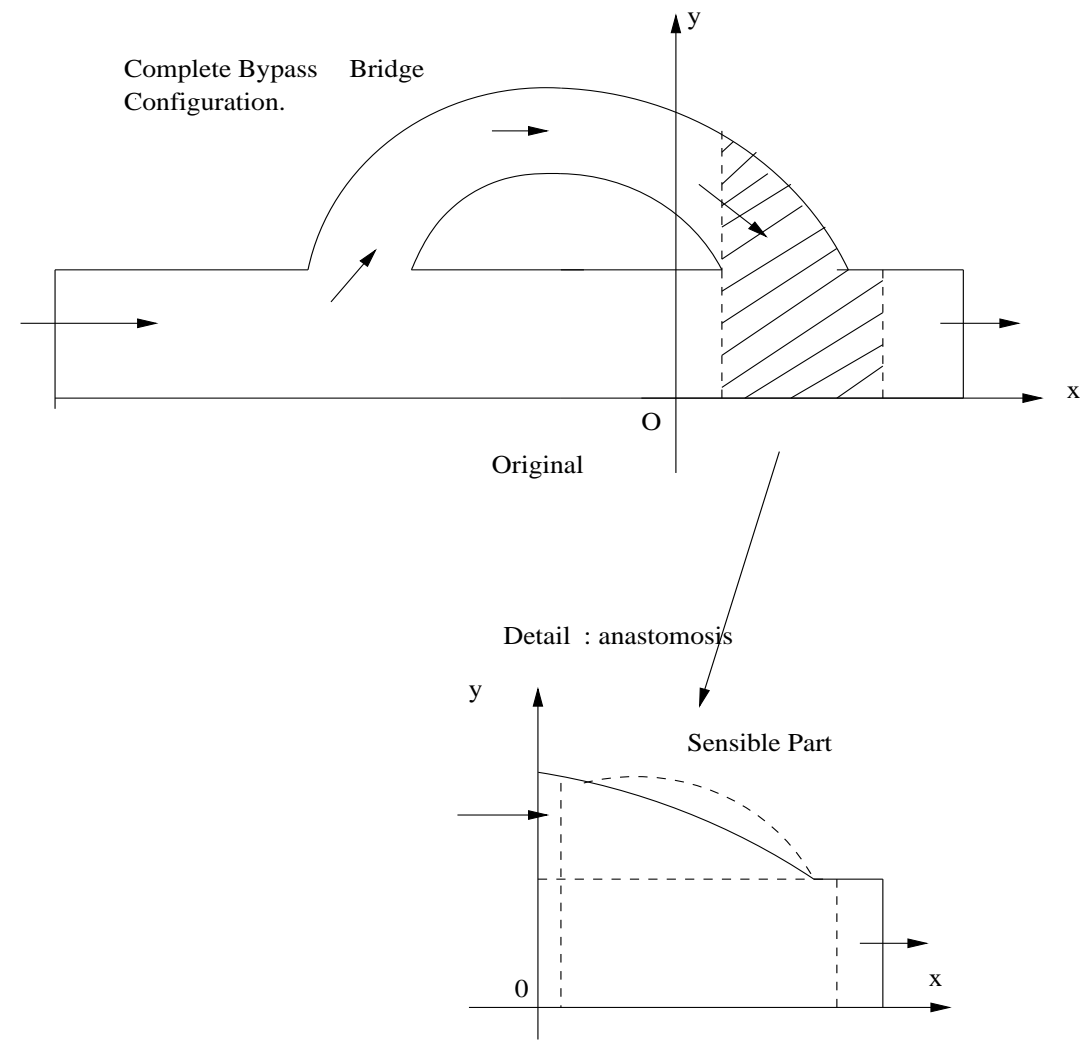

Figure 1: Idealized, 2-D bypass bridge configuration (top) and detail of the sensible part for the optimization process .

find $\underline{v}, p$ s.t.

$$
\left\{\begin{array}{l}
-\nu \Delta \underline{v}+\nabla p=\underline{F} \text { in } \Omega, \\
\nabla \cdot \underline{v}=0 \text { in } \Omega, \\
\underline{v}=\underline{v}_{\text {in }} \text { on } \Gamma_{\text {in }}, \underline{v}=0 \text { on } \Gamma_{w_{1}} \cup \Gamma_{w_{3}}, \\
-p \cdot \underline{n}+\nu \frac{\partial \underline{v}}{\partial n}=\underline{g}_{\text {out }} \text { on } \Gamma_{\text {out }} \cup \Gamma_{w_{2}},
\end{array}\right.
$$

where $\underline{n}=\left(n_{1}, n_{2}\right)$ is the outward unit normal vector on $\Gamma \equiv \partial \Omega$. In the sequel: $\left.\tau=\left(n_{2},-n_{1}\right)\right), \underline{F}=\underline{F}(x, y), \underline{v}_{f}=\underline{v}_{f}(x, y), \underline{g}_{\text {out }}=\underline{g}_{\text {out }}(x, y)$ are given 


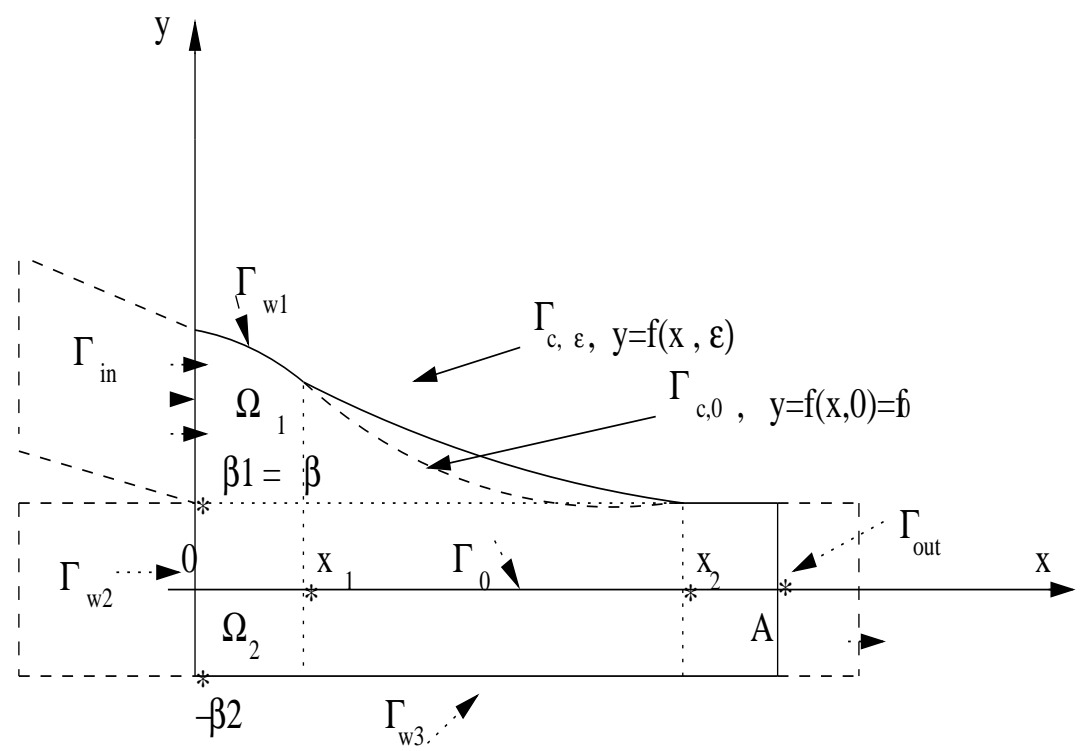

Figure 2: $\bar{\Omega}=\bar{\Omega}_{1} \cup \bar{\Omega}_{2}, \Gamma_{w}=\Gamma_{w_{1}} \cup \Gamma_{w_{2}} \cup \Gamma_{w_{3}}, \Gamma_{0}=\bar{\Omega}_{1} \cup \bar{\Omega}_{1}$.

vector function, $\nu=$ const $>0$ and $v_{f}=\left\{\underline{v}_{\text {in }}\right.$ on $\Gamma_{i n} ; \underline{0}$ on $\left.\Gamma_{w_{1}} \cup \Gamma_{w_{3}}\right\}$. In the following we will impose some additional restriction to $p$ (for example $\int_{\Omega} p d \Omega=0$ or others).

The subset $\Gamma_{c, \varepsilon}$ of $\Gamma_{w_{1}}$ is parametrized by a function $f(x, \varepsilon)$ of $\underline{x} \in\left[x_{1}, x_{2}\right]$ and of small parameter $\varepsilon \in\left[-\varepsilon_{0}, \varepsilon_{0}\right], \varepsilon_{0}=$ const. More precisely we assume that $f(x, \varepsilon)$ can be developed as follows:

$$
f(x, \varepsilon)=f_{0}(x)+\varepsilon f_{1}(x)+\varepsilon^{2} f_{2}(x)+\ldots,
$$

where $f_{k} \in \mathbb{W}_{\infty}^{1}\left(x_{1}, x_{2}\right), k \geq 0$ and $f_{k}\left(x_{1}\right)=f_{k}\left(x_{2}\right)=0, k \geq 1$. Here the function $f_{0}(x)>0$ describes the original subset $\Gamma_{c, 0}$ of the "unperturbed domain" $\Gamma_{0} \equiv \partial \Omega_{0}$ of the domain $\Omega_{0}$ (see Fig. (3)), while $f_{k}(x), k \geq 1$, could be unknown in our application to control problem (see Section 4).

The weak statement of $(1)$ reads: find $\underline{v} \in\left(\mathbb{H}^{1}(\Omega)\right)^{2}, p \in \mathbb{L}^{2}(\Omega)$ s.t.

$$
\left\{\begin{array}{l}
a(\underline{v}, \underline{\hat{v}})=b(p, \underline{\hat{v}})+G(\underline{\hat{v}}) \forall \underline{\hat{v}} \in \mathbb{X}, \\
b(\hat{\hat{p}}, \underline{v})=0 \forall \forall \in \mathbb{L}^{2}(\Omega), \\
\underline{v}=\underline{v}_{f} \text { on } \Gamma_{i n} \cup \Gamma_{w_{1}} \cup \Gamma_{w_{3}},
\end{array}\right.
$$

where

$$
\begin{gathered}
a(\underline{v}, \underline{\hat{v}})=\int_{\Omega} \nu \nabla \underline{v} \cdot \nabla \underline{\hat{v}} d \Omega \quad(d \Omega=d x d y) \\
b(p, \underline{\hat{v}})=\int_{\Omega} p \nabla \cdot \underline{\hat{v}} d \Omega, G(\underline{\hat{v}})=\int_{\Omega} \underline{F} \cdot \underline{\hat{v}} d \Omega+\int_{\Gamma_{\text {out }} \cup \Gamma_{w_{2}}} \underline{g}_{\text {out }} \cdot \underline{\hat{v}} d \Gamma, \\
\mathbb{X} \equiv\left\{\underline{\hat{v}}: \underline{\hat{v}} \in\left(\mathbb{H}^{1}(\Omega)\right)^{2}, \underline{\hat{v}}=0 \text { on } \Gamma_{\text {in }} \cup \Gamma_{w_{1}} \cup \Gamma_{w_{3}} \cdot\right\}
\end{gathered}
$$

Here we emphasize the dependence of $a(.,),. b(.,$.$) and G($.$) on f$ - a parametrization of the part $\Gamma_{c, \varepsilon}$ of the domain boundary. However, this dependence will be understood for simplicity of notations. 


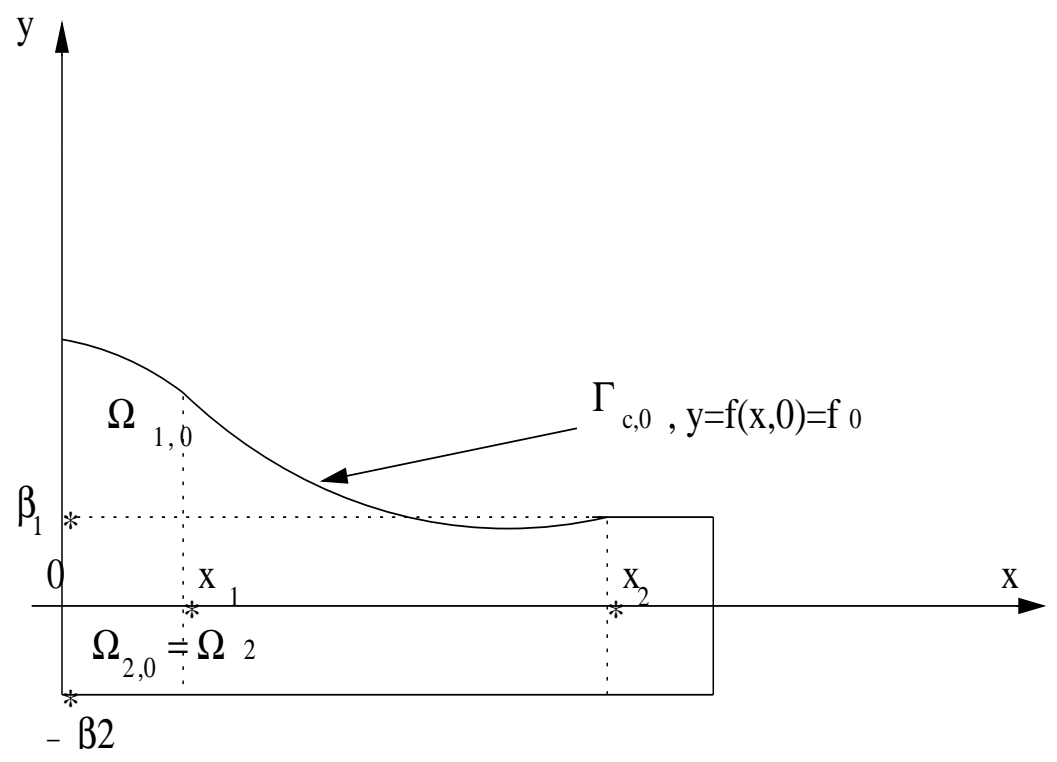

Figure 3: "Unperturbed domain" $\Omega_{0}, \bar{\Omega}_{0}=\bar{\Omega}_{0_{1}} \cup \bar{\Omega}_{0_{2}}$.

\section{The Problem for the perturbation functions}

Assume that $f(x, \varepsilon)>0$ and consider the following variables transformation:

$$
\mathrm{T}_{f}: \bar{\Omega}_{1} \cup \bar{\Omega}_{2} \rightarrow \overline{\tilde{\Omega}}, \underline{\tilde{x}}=\mathrm{T}_{f}(\underline{x}),
$$

which is given as follows

$$
\begin{cases}\tilde{x}=x, \tilde{y}=\frac{\beta}{f(x, \varepsilon)} y & \text { if } \underline{x}=(x, y) \in \Omega_{1} \\ \tilde{x}=x, \tilde{y}=y & \text { if } \underline{x}=(x, y) \in \Omega_{2}\end{cases}
$$

where $\tilde{\Omega} \equiv \tilde{\Omega}_{\text {simp }}=\left\{\underline{\tilde{x}}=(\tilde{x}, \tilde{y}): 0<\tilde{x}<A,-\beta_{2}<Y<\beta_{1} \equiv \beta\right\}, \tilde{\Omega}_{1}=\tilde{\Omega}_{1_{\text {simp }}}$, $\tilde{\Omega}_{2}=\tilde{\Omega}_{2_{\text {simp }}}(\operatorname{see} \operatorname{Fig}(4))$.

We set

$$
\underline{\tilde{v}}(\tilde{x}, \tilde{y}) \equiv \underline{v} \circ \mathrm{T}_{f}^{-1}(\underline{\tilde{x}})=\underline{v}(\tilde{x}, \tilde{y} f(\tilde{x}, \varepsilon) / \beta) .
$$

where $\underline{\tilde{v}}=(\tilde{u}, \tilde{v})$. Then,

$$
d x d y=\frac{f(\tilde{x}, \epsilon)}{\beta} d \tilde{x} d \tilde{y}
$$

and the following relations hold on the derivatives of a function $\phi$ and $\underline{v}$ :

$$
\begin{gathered}
\left\{\begin{array}{l}
\left(\frac{\partial \phi}{\partial y}\right)(\tilde{x}, \tilde{y})=\frac{\beta}{f(\tilde{x}, \varepsilon)} \frac{\partial \tilde{\phi}(\tilde{x}, \tilde{y})}{\partial \tilde{y}}, \\
\left(\frac{\partial \phi}{\partial y}\right)(\tilde{x}, \tilde{y})=\frac{\partial \phi(\tilde{x}, \tilde{y})}{\partial \tilde{x}}-\tilde{y} \frac{f_{x}(\tilde{x}, \varepsilon)}{f(\tilde{x}, \varepsilon)} \frac{\partial \tilde{\phi}(\tilde{x}, \tilde{y})}{\partial \tilde{y}}\left(w i t h f_{x} \equiv \frac{d f}{d x}\right)
\end{array}\right. \\
\left\{\begin{array}{l}
\tilde{\mathcal{D}}(f) \underline{\tilde{v}}(\tilde{x}, \tilde{y}) \equiv\left((\nabla \cdot \underline{v}) \circ \mathrm{T}_{f}^{-1}\right)(\tilde{x}, \tilde{y})=\frac{\partial \tilde{u}}{\partial \tilde{x}}-\tilde{y} \frac{f_{x}(\tilde{x}, \epsilon)}{f(\tilde{x},)} \frac{\partial \tilde{u}}{\partial \tilde{y}}+\frac{\beta}{f(\tilde{x}, \varepsilon)} \frac{\partial \tilde{v}}{\partial \tilde{y}}, \\
\tilde{\mathcal{R}}(f) \underline{\tilde{v}}(\tilde{x}, \tilde{y}) \equiv\left((\nabla \times \underline{v}) \circ \mathrm{T}_{f}^{-1}\right)(\tilde{x}, \tilde{y})=\frac{\partial \tilde{v}}{\partial \tilde{x}}-\tilde{y} \frac{f_{x}(\tilde{x}, \epsilon)}{f(\tilde{x}, \varepsilon)} \frac{\partial \tilde{v}}{\partial \tilde{y}}-\frac{\beta}{f(\tilde{x}, \varepsilon)} \frac{\partial \tilde{u}}{\partial \tilde{y}},
\end{array}\right.
\end{gathered}
$$




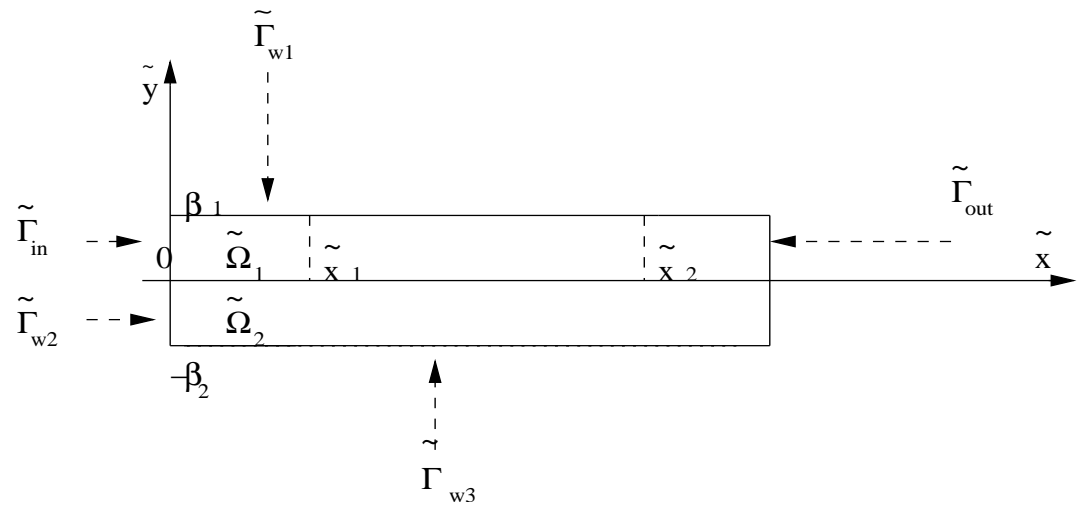

Figure 4: The "simple" domain $\tilde{\Omega}_{\text {simp }}$.

So, in $\tilde{\Omega}$ we have:

$$
\tilde{\mathcal{D}}(f) \underline{\tilde{v}}=m_{2} \tilde{\nabla} \cdot \underline{\tilde{v}}+m_{1} \tilde{\mathcal{D}}(f) \underline{\tilde{v}}, \tilde{\mathcal{R}}(f) \underline{\tilde{v}}=m_{2} \tilde{\nabla} \times \underline{\tilde{v}}+m_{1} \tilde{\mathcal{R}}(f) \underline{\tilde{v}} ;
$$

where $\tilde{\nabla} \phi \equiv\left(\frac{\partial \phi}{\partial \tilde{x}}, \frac{\partial \phi}{\partial \tilde{y}}\right)$, while $m_{s}$ is the characteristic function of $\Omega_{s}(s=1,2, \ldots)$. To simplify the notations from now on we will set (unless otherwise specified):

$\underline{\tilde{x}}=\underline{x}, \underline{\tilde{v}}(\tilde{x}, \tilde{y}) \equiv \underline{v}(x, y), \tilde{u}=u, \tilde{v}=v, \ldots, \tilde{\mathcal{D}}=\mathcal{D}, \tilde{\mathcal{R}}=\mathcal{R}, \tilde{\Omega}_{s i m p} \equiv \Omega_{s i m p}, \tilde{\Gamma}_{w_{k}} \equiv \Gamma_{w_{k}}$.

Then the problem (3) in the new variables reads as follows:

$$
\left\{\begin{array}{l}
a(f ; \underline{v}, \underline{\hat{v}})=b(f ; p, \underline{\hat{v}})+G(f ; \underline{\hat{v}}) \forall \underline{\hat{v}} \in \mathbb{X} \\
b(f ; \underline{\hat{p}}, \underline{v})=0 \forall \hat{p} \in \mathbb{L}^{2}\left(\Omega_{\text {simp }}\right), \\
\underline{v}=\underline{v}_{f} \text { on } \Gamma_{i n} \cup \Gamma_{w_{1}} \cup \Gamma_{w_{3}},
\end{array}\right.
$$

where

$$
\mathbb{X} \equiv\left\{\underline{v}: \underline{v} \in\left(\mathbb{H}^{1}\left(\Omega_{\text {simp }}\right)\right)^{2}, \underline{v}=0 \text { on } \Gamma_{i n} \cup \Gamma_{w_{1}} \cup \Gamma_{w_{3}} .\right\}
$$

Here we emphasize the dependence of $a(f ; .,),. b(f ; .,$.$) , and G(f ;$.$) on f$. Precisely, (with $\Omega_{1} \equiv \tilde{\Omega}_{1}, \Omega_{2} \equiv \tilde{\Omega}_{2}$ ):

$$
\begin{gathered}
a(f ; \underline{v}, \underline{\hat{v}})=a_{1}(f ; \underline{v}, \underline{\hat{v}})+a_{2}(\underline{v}, \underline{\hat{v}}) \\
a_{1}(f ; \underline{v}, \underline{\hat{v}})=\int_{\Omega_{1}} \frac{f \nu}{\beta}\left(\left(\frac{\partial \underline{v}}{\partial x}-\frac{y f_{x}}{f} \frac{\partial \underline{v}}{\partial y}\right) \cdot\left(\frac{\partial \underline{\hat{v}}}{\partial x}-\frac{y f_{x}}{f} \frac{\partial \underline{\hat{v}}}{\partial y}\right)+\frac{\beta^{2}}{f^{2}} \frac{\partial \underline{v}}{\partial y} \cdot \frac{\partial \underline{\hat{v}}}{\partial y}\right) d x d y \\
a_{2}(\underline{v}, \underline{\hat{v}})=\int_{\Omega_{2}} \nu\left(\frac{\partial \underline{v}}{\partial x} \cdot \frac{\partial \underline{\hat{v}}}{\partial x}+\frac{\partial \underline{v}}{\partial y} \cdot \frac{\partial \underline{\hat{v}}}{\partial y}\right) d x d y . \\
b(f ; p, \underline{\hat{v}})=b_{1}(f ; p, \underline{\hat{v}})+b_{2}(p, \underline{\hat{v}}), \\
b_{1}(f ; p, \underline{\hat{v}})=\int_{\Omega_{1}} \frac{f}{\beta} \mathcal{D}(f) \underline{\hat{v}} d x d y, \quad b_{2}(p, \underline{\hat{v}})=\int_{\Omega_{2}} p \nabla \cdot \underline{\hat{v}} d x d y, \\
G(f ; \underline{\hat{v}})=G_{1}(f ; \underline{\hat{v}})+G_{2}(\underline{\hat{v}}), \\
G_{1}(f ; \underline{\hat{v}})=\int_{\Omega_{1}} \frac{f}{\beta} \underline{F} \cdot \underline{\hat{v}} d x d y+\int_{\Gamma_{\text {out }} \cap \partial \Omega_{1}}{ }_{g} \text { out } \\
\end{gathered}
$$




$$
G_{2}(\underline{\hat{v}})=\int_{\Omega_{2}} \underline{F} \cdot \underline{\hat{v}} d x d y+\int_{\Gamma_{\text {out }} \cap \partial \Omega_{2}} \underline{g}_{\text {out }} \cdot \underline{\hat{v}} d \Gamma .
$$

Note that the functions $\underline{\hat{v}}, \tilde{p}$ on (7) can be assumed to be indipendent of $\varepsilon$ in the sequel.

Assume that the problem (7) has a solution $\underline{v}, p$ that is infinitely differentiable with respect to $\varepsilon$ :

$$
\left\{\begin{array}{l}
\underline{v}=\underline{v}_{0}+\varepsilon \underline{v}_{1}+\varepsilon^{2} \underline{v}_{2}+\ldots \\
p=p_{0}+\varepsilon p_{1}+\varepsilon^{2} p_{2}+\ldots
\end{array}\right.
$$

where $p_{k} \in \mathbb{L}^{2}, \underline{v}_{k} \in \mathbb{X}, k \geq 1$. Using (2), (8) and small perturbation techniques we can derive the equations to $\underline{v}_{k}, p_{k}, k \geq 0$. Consider these equations only for $k=0$ and $k=1$.

$\underline{\mathrm{k}=0}$ :

$$
\left\{\begin{array}{l}
a\left(f_{0} ; \underline{v}_{0}, \underline{\hat{v}}\right)=b\left(f_{0} ; p_{0}, \underline{\hat{v}}\right)+G\left(f_{0} ; \underline{\hat{v}}\right) \forall \underline{\hat{v}} \in \mathbb{X}, \\
b\left(f_{0} ; \underline{\hat{p}}, \underline{v}_{0}\right)=0 \forall \hat{p} \in \mathbb{L}^{2}(\Omega), \\
\underline{v}_{0}=\underline{v}_{f} \text { on } \Gamma_{i n} \cup \Gamma_{w_{1}} \cup \Gamma_{w_{3}} .
\end{array}\right.
$$

Correspondingly we define:

$$
\mathcal{R}_{o b s, 0} \equiv \mathcal{R}\left(f_{0}\right) \underline{v}_{0}
$$

$\underline{\mathrm{k}=1}:$

$$
\left\{\begin{array}{l}
a\left(f_{0} ; \underline{v}_{1}, \underline{\hat{v}}\right)=b\left(f_{0} ; p_{1}, \underline{\hat{v}}\right)+\left.\frac{\partial}{\partial \varepsilon} b\left(f ; p_{0}, \underline{\hat{v}}\right)\right|_{\varepsilon=0}+\left.\frac{\partial}{\partial \varepsilon} G(f ; \underline{\hat{v}})\right|_{\varepsilon=0}+ \\
-\left.\frac{\partial}{\partial \varepsilon} a\left(f ; \underline{v}_{0}, \underline{\hat{v}}\right)\right|_{\varepsilon=0} \forall \underline{\hat{v}} \in \mathbb{X}, \\
b\left(f_{0} ; \underline{\hat{p}}, \underline{v}_{1}\right)+\left.\frac{\partial}{\partial \varepsilon} b\left(f ; \hat{p}, \underline{v}_{0}\right)\right|_{\varepsilon=0}=0 \forall \hat{p} \in \mathbb{L}^{2}(\Omega), \\
\underline{v}_{1}=0 \text { on } \Gamma_{\text {in }} \cup \Gamma_{w_{1}} \cup \Gamma_{w_{3}},
\end{array}\right.
$$

where

$$
\begin{gathered}
\left.\frac{\partial}{\partial \varepsilon} b\left(f ; p_{0}, \underline{\hat{v}}\right)\right|_{\varepsilon=0} \equiv b_{f}\left(f_{1}, p_{0}, \underline{\hat{v}}\right)=\int_{\Omega_{1}} \frac{f_{1}}{\beta} p_{0} \mathcal{D}\left(f_{0}\right) \underline{\hat{v}} d x d y+\int_{\Omega_{1}} \frac{f_{0}}{\beta} p_{0} \mathcal{D}_{f}\left(f_{1}, \underline{\hat{v}}\right) d x d y \\
\left.\mathcal{D}_{f}\left(f_{1}, \underline{\hat{v}}\right) \equiv \frac{\partial}{\partial \varepsilon} \mathcal{D}(f) \underline{\hat{v}}\right|_{\varepsilon=0}=-\left[y\left(\frac{f_{1, x} f_{0}-f_{0, x} f 1}{f_{0}^{2}}\right) \frac{\partial \hat{u}}{\partial y}+\frac{\beta f_{1}}{f_{0}^{2}} \frac{\partial \hat{v}}{\partial y}\right] \\
\left.\mathcal{D}_{f}\left(f_{1}, \underline{v}_{0}\right) \equiv \frac{\partial}{\partial \varepsilon} \mathcal{D}(f) \underline{v}_{0}\right|_{\varepsilon=0}\left(\equiv \mathcal{D}_{f} f_{1} \text { for the sequel }\right), \\
\left.\frac{\partial}{\partial \varepsilon} G(f ; \underline{\hat{v}})\right|_{\varepsilon=0} \equiv G_{1}\left(f_{1} ; \underline{\hat{v}}\right)=\int_{\Omega_{1}} \frac{f_{1}}{\beta} F \cdot \underline{\hat{v}} d x d y, \\
\left.\frac{\partial}{\partial \varepsilon} a\left(f ; \underline{v}_{0}, \underline{\hat{v}}\right)\right|_{\varepsilon=0} \equiv a_{f}\left(f_{1} ; \underline{v}_{0}, \underline{\hat{v}}\right)=\int_{\Omega_{1}} \frac{f_{1} \nu}{\beta}\left(\left(\frac{\partial \underline{v}}{\partial x}-\frac{y f_{0, x}}{f_{0}} \frac{\partial \underline{v_{0}}}{\partial y}\right) \cdot\left(\frac{\partial \underline{\hat{v}}}{\partial x}-\frac{y f_{0, x}}{f_{0}} \frac{\partial \underline{\hat{v}}}{\partial y}\right)+\frac{\beta^{2}}{f_{0}^{2}} \frac{\partial \underline{\underline{v}}}{\partial y} \cdot \frac{\partial \underline{\hat{v}}}{\partial y}\right) d x d y+ \\
-\int_{\Omega_{1}} \frac{f_{0} \nu}{\beta} y \frac{\left(f_{1, x} f_{0}-f_{0, x} f_{1}\right)}{f_{0}^{2}}\left(\frac{\underline{v} 0}{\partial y} \cdot\left(\frac{\partial \underline{\hat{v}}}{\partial x}-\frac{y f_{0, x}}{f_{0}} \frac{\partial \underline{\hat{v}}}{\partial y}\right)+\left(\frac{\partial \underline{v}_{0}}{\partial x}-\frac{y f_{0, x}}{f_{0}} \frac{\partial \underline{v}_{0}}{\partial y}\right) \cdot \frac{\partial \underline{\hat{v}}}{\partial y}\right) d x d y \\
-\int_{\Omega_{1}} \frac{f_{0} \nu}{\beta}\left(\frac{2 \beta^{2} f_{1}}{f_{0}^{3}}\right) \frac{\partial \underline{v}_{0}}{\partial y} \cdot \frac{\partial \underline{\hat{v}}}{\partial y} d x d y .
\end{gathered}
$$

So the problem for $\underline{v}_{1}, p_{1}$ reads as follows: find $\underline{v}_{1} \in \mathbb{X}, p_{1} \in \mathbb{L}^{2}(\Omega)$ s.t.:

$$
\left\{\begin{array}{l}
a\left(f_{0} ; \underline{v}_{1}, \underline{\hat{v}}\right)-b\left(f_{0} ; p_{1}, \underline{\hat{v}}\right)=b_{f}\left(f_{1} ; p_{0}, \underline{\hat{v}}\right)+G_{1}\left(f_{1} ; \underline{\hat{v}}\right)-a_{f}\left(f_{1} ; \underline{v}_{0}, \underline{\hat{v}}\right) \forall \underline{\hat{v}} \in \mathbb{X}, \\
b\left(f_{0} ; \underline{\hat{p}}_{1}\right)+b_{f}\left(f_{1} ; \hat{p}, \underline{v}_{0}\right)=0 \forall \hat{p} \in \mathbb{L}^{2}(\Omega),
\end{array}\right.
$$


This is a generalized Stokes Problem. By a similar technique we can derive the equations for $\underline{v}_{k}, p_{k}$ with $k \geq 2$. However we will not proceed further this development in these notes.

\section{The Shape Optimization Problem}

Suppose now that the function $f_{1}(x)$ in (11) is unknown as well as $\underline{v}_{1}, p_{1}$ in (11). To complete problem (11) we will have to formulate some "additional equations"; otherwise we should require that $f_{1}$ be determined by minimizing a suitable "cost functional".

Problem (3) can be supplemented by the "additional equations":

$$
\mathcal{C}(f, \underline{v}, p)=0
$$

where $\mathcal{C}$ is an operator(linear or nonlinear) defined on $\mathbb{H}_{0}^{1}\left(x_{1}, x_{2}\right) \times \mathbb{X} \times \mathbb{L}^{2}(\Omega)$. We assume $\mathcal{C}$ to be smooth with respect to its variables $f, \underline{v}, p$. Using representations $(2),(8)$ we derive from (13) the following equation:

$$
\mathcal{C}(f, \underline{v}, p)=\mathcal{C}\left(f_{0}, \underline{v}_{0}, p_{0}\right)+\varepsilon \mathcal{C}_{1}\left(f_{1}, \underline{v}_{1}, p_{1}\right)+\mathcal{O}\left(\varepsilon^{2}\right)=0, \quad \forall \varepsilon \in\left[-\varepsilon_{0}, \varepsilon_{0}\right]
$$

where

$$
\left.\mathcal{C}_{1}\left(f_{1}, \underline{v}_{1}, p_{1}\right) \equiv \frac{\partial \mathcal{C}}{\partial \varepsilon}(f, \underline{v}, p)\right|_{\varepsilon=0} .
$$

If we assume that the data of our problems are such that $\mathcal{C}\left(f_{0}, \underline{v}_{0}, p_{0}\right)=0$, then we can use

$$
\mathcal{C}_{1}\left(f_{1}, \underline{v}_{1}, p_{1}\right)=0
$$

as the "additional equation" to complete (11). An alternative approach would consist in replacing the exact controllability equation (16) by the following minimization problem:

$$
\inf _{f_{1}}=\int_{\Omega_{\text {simp }}} \frac{f_{0}}{\beta}\left|\mathcal{C}_{1}\left(f_{1}, \underline{v}_{1}, p_{1}\right)\right|^{2} d x d y
$$

where we assume $\mathcal{C}_{1}$ has image in $\mathbb{L}^{2}(\Omega)$. Note, that (17) is one of weak statement of (16).

In the next sections we apply the approach described above for the completion of (11) and we will use the following special choice of (13):

$$
\mathcal{C}(f, \underline{v}) \equiv\left((\nabla \times \underline{v}) \circ \mathrm{T}_{f}^{-1}\right)(x, y)-\mathcal{R}_{o b s, \varepsilon}(x, y) \text { in } \Omega_{w d} \subseteq \Omega_{s i m p},
$$

where $\Omega_{w d}$ is a suitable subset of $\Omega_{s i m p}$ in which we want our "additional equation " (or our "control") to take place. We assume in (18) that

$\mathcal{R}_{o b s, \varepsilon}=\mathcal{R}_{o b s, 0}+\varepsilon \mathcal{R}_{o b s, 1}+\varepsilon^{2} \mathcal{R}_{o b s, 2}+\ldots, \mathcal{R}_{o b s, 0}={ }^{d e f}\left(\left(\nabla \times \underline{v}_{0}\right) \circ \mathrm{T}_{f_{0}}^{-1}\right)(x, y)$.

Then we have: $\mathcal{C}\left(f_{0}, \underline{v}_{0}\right)=0$, while the equation (16) reads:

$$
\mathcal{C}\left(f_{1}, \underline{v}_{1}\right)=\mathcal{R}\left(f_{0}\right) \underline{v}_{1}+m_{1} \mathcal{R}_{f} f_{1}-\mathcal{R}_{o b s, 1}=0 \text { in } \Omega_{w d},
$$

where

$$
\mathcal{R}\left(f_{0}\right) \underline{v}_{1}=\left(\nabla \times \underline{v}_{1}\right) \circ \mathrm{T}_{f_{0}}^{-1}(x, y)=\frac{\partial v_{1}}{\partial x}-\frac{y f_{0, x}}{f_{0}} \frac{\partial v_{1}}{\partial y}-\frac{\beta}{f_{0}} \frac{\partial u_{1}}{\partial y},
$$




$$
\mathcal{R}_{f} f_{1} \equiv \mathcal{R}_{f}\left(f_{1}, \underline{v}_{0}\right)=-y \frac{\left(f_{1, x} f_{0}-f_{0, x} f_{1}\right)}{f_{0}^{2}} \frac{\partial v_{0}}{\partial y}+\frac{\beta f_{1}}{f_{0}^{2}} \frac{\partial u_{0}}{\partial y} .
$$

Therefore we have the problem: find $\underline{v}_{1} \in \mathbb{X}, p_{1} \in \mathbb{L}^{2}(\Omega), f_{1} \in \mathbb{H}_{0}^{1}\left(x_{1}, x_{2}\right)$ s.t.

$$
\left\{\begin{array}{l}
a\left(f_{0} ; \underline{v}_{1}, \underline{\hat{v}}\right)=b\left(f_{0} ; p_{1}, \underline{\hat{v}}\right)+b_{f}\left(f_{1} ; p_{0}, \hat{\underline{v}}\right)+G_{1}\left(f_{1} ; \underline{\hat{v}}\right)-a_{f}\left(f_{1} ; \underline{v}_{0}, \underline{\hat{v}}\right) \forall \underline{\hat{v}} \in \mathbb{X}, \\
b\left(f_{0} ; \underline{\hat{p}}, \underline{v}_{1}\right)+b_{f}\left(f_{1} ; \hat{p}, \underline{v}_{0}\right)=0 \forall \forall \hat{p} \in \mathbb{L}^{2}(\Omega), \\
\mathcal{R}\left(f_{0}\right) \underline{\underline{v}}_{1}+m_{1} \mathcal{R}_{f} f_{1}-\mathcal{R}_{o b s, 1}=0 \text { in } \Omega_{w d},
\end{array}\right.
$$

where $\mathcal{R}_{o b s, 1}$ is a given function. Problem (21) is an "exact controllability problem". These problems have solutions in some particular cases only. Therefore we replace (21) by the following optimal control problem: find $\underline{v}_{1} \in \mathbb{X}, p_{1} \in \mathbb{L}^{2}(\Omega)$, $f_{1} \in \mathbb{H}_{0}^{1}\left(x_{1}, x_{2}\right)$ s.t.

$$
\left\{\begin{array}{l}
a\left(f_{0} ; \underline{v}_{1}, \underline{\hat{v}}\right)-b\left(f_{0} ; p_{1}, \underline{\hat{v}}\right)=b_{f}\left(f_{1} ; p_{0}, \underline{\hat{v}}\right)+G_{1}\left(f_{1} ; \underline{\hat{v}}\right)-a_{f}\left(f_{1} ; \underline{v}_{0}, \underline{\hat{v}}\right) \forall \underline{\hat{v}} \in \mathbb{X}, \\
b\left(f_{0} ; \underline{\hat{p}}, \underline{v}_{1}\right)+b_{f}\left(f_{1} ; \hat{p}, \underline{v}_{0}\right)=0 \forall \hat{p} \in \mathbb{L}^{2}(\Omega), \\
\inf _{f_{1}}=\frac{\alpha}{2}\|f\|_{\mathbb{H}_{0}^{1}\left(x_{1}, x_{2}\right)}^{2}+\gamma_{1} J_{1}\left(f_{1}, \underline{v}_{1}\right),
\end{array}\right.
$$

where

$$
J_{1}\left(f_{1}, \underline{v}_{1}\right)=\frac{1}{2} \int_{\Omega} m_{w d} \frac{f_{0}}{\beta}\left|\mathcal{R}\left(f_{0}\right) \underline{v}_{1}+m_{1} \mathcal{R}_{f} f_{1}-\mathcal{R}_{o b s, 1}\right|^{2} d x d y,
$$

$\alpha=$ const $\geq 0$ is a small regularization parameter, $\gamma_{1}>0$ is a weight coefficient, $m_{w d}$ is the characteristic function of $\Omega_{w d}$.

If $\alpha=0$ (22) provides the weak statement of problem (21). Otherwise the solution $v_{1}=v_{1}(\alpha), p_{1}=p_{1}(\alpha), f_{1}=f_{1}(\alpha)$ of (22) represents an approximate solution of (21).

We will also consider a generalized optimal control problem still given by (22) however now instead of $J_{1}$ we use

$$
J\left(f_{1}, \underline{v}_{1}, p_{1}\right)=\gamma_{1} J_{1}\left(f_{1}, \underline{v}_{1}\right)+\gamma_{2} J_{2}\left(f_{1}, \underline{v}_{1}, p_{1}\right) .
$$

Here $\gamma_{2}=$ const $\geq 0$ is a weight coefficient, $J_{2}\left(f_{1}, \underline{v}_{1}, p_{1}\right)$ is an additional functional assumed to be quadratic. Some examples of $J_{2}\left(f_{1}, \underline{v}_{1}, p_{1}\right)$ follow.

EXAMPLE 1. For instance

$$
J_{2}\left(f_{1}, \underline{v}_{1}, p_{1}\right) \equiv J_{2}\left(\underline{v}_{1}, p_{1}\right)=\frac{1}{2}\left(|| p-p_{\text {out }} \|_{\mathbb{L}^{2}\left(\Gamma_{\text {out }}\right)}^{2}+\int_{\Gamma_{\text {out }}}\left|\underline{v}-\underline{v}_{\text {out }}\right|^{2} d \Gamma\right)
$$

where $p_{\text {out }}, \underline{v}_{\text {out }}$ are given.

EXAMPLE 2. Now let $\Omega_{o b}$ be a "small" subdomain of $\Omega$ and $\Gamma_{w_{3}} \subset \partial \Omega_{o b} \equiv$ $\Gamma_{o b}$ :

$$
\Omega_{o b}=(0, A) \times\left(-\beta_{2},-\beta_{2}+\delta\right), \text { with } 0<\delta \ll \beta_{1}
$$

If $\Omega=\Omega_{1}$ (i.e. $\beta_{2}=0$ ), we can take

$$
J_{2}\left(f_{1}, \underline{v}_{1}, p_{1}\right) \equiv J_{2}\left(\underline{v}_{1}\right)=\frac{1}{2} \int_{\Omega_{o b}}\left|\underline{v}-\underline{v}_{o b s}\right|^{2} \frac{f_{0}}{\beta} d x d y,
$$

where $\underline{v}_{o b s}$ is a prescribed vector-function.

EXAMPLE 3. Consider the following case: $\Omega_{1}=\Omega, \int_{\Omega} p_{1} d \Omega=0$,

$$
J_{2}\left(f_{1}, \underline{v}_{1}, p_{1}\right) \equiv J_{2}\left(\underline{v}_{1}\right)=\frac{1}{2} \int_{\Gamma_{w_{3}}}\left|\frac{\partial \underline{v}_{1}}{\partial n}-\underline{g}_{1, o b s}\right|^{2} d \Gamma,
$$




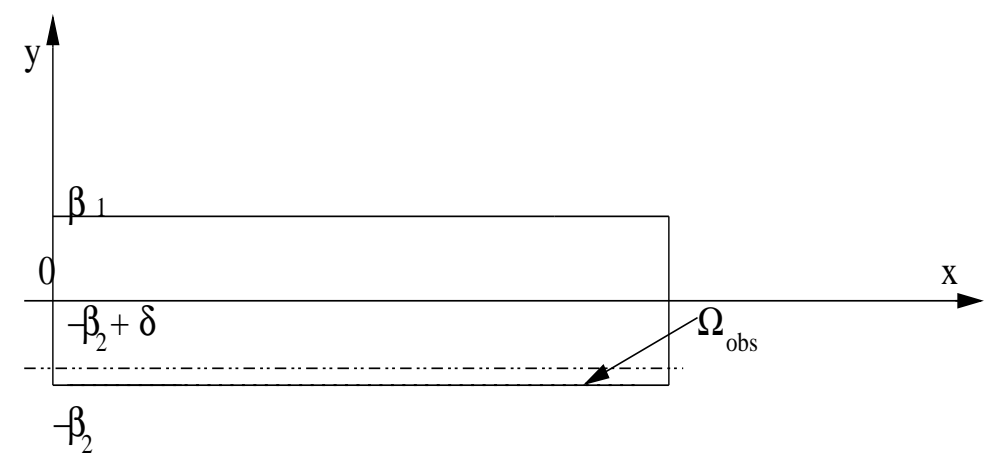

Figure 5: Subdomain $\Omega_{o b}$.

with a given $\underline{g}_{1, o b s}$.

EXAMPLE 4. Finally we consider:

$$
J_{2}\left(f_{1}, \underline{v}_{1}, p_{1}\right) \equiv J_{2}\left(\underline{v}_{1}\right)=\frac{1}{2} \int_{\Omega_{o b s}} \frac{f_{0}}{\beta}\left|\frac{\beta}{f_{0}} \frac{\partial \underline{v}_{1}}{\partial y}-\underline{g}_{2, o b s}\right|^{2} d \Omega,
$$

with a given $\underline{g}_{2, o b s}$.

REMARK. The term $J_{2}$ can be considered as an "overdetermination" of the problem. Indeed, it allows in principle the control of further quantities of physical interest. Using such a $J_{2}$ makes easier to prove uniqueness but more troublesome to analyze existence for the optimal control problem.

\section{The variational equations of the optimal con- trol problem}

While considering (22) we can continue to work in the simple domain $\Omega_{\text {simp }}$. Another possibility consists of using the new variables transformation

$$
T_{f_{0}}^{-1}(\underline{\tilde{x}})=\underline{x}, \underline{\tilde{x}} \in \Omega_{s i m p}, \underline{x} \in \Omega_{0},
$$

given by

$$
\left\{\begin{array}{l}
x=\tilde{x}, y=\frac{f_{0}(\tilde{x})}{\beta} \tilde{y} \text { if } \underline{\tilde{x}}=(\tilde{x}, \tilde{y}) \in \tilde{\Omega}_{1} \subset \Omega_{\text {simp }} \\
x=\tilde{x}, y=\tilde{y} \quad \text { if } \underline{\tilde{x}}=(\tilde{x}, \tilde{y}) \in \tilde{\Omega}_{2} \subset \Omega_{\text {simp }}
\end{array}\right.
$$

After applying (27) we will work in the "unperturbed" domain $\Omega_{0}$ (see Fig. 6) and the expressions of bilinear forms from (22) will be more simple. Let us use the variable transformation $(27)$. Then the problem (22) reads: find $\underline{v} \equiv \underline{v}_{1}$, $p \equiv p_{1}, f \equiv f_{1}{ }^{1}$

$$
\left\{\begin{array}{l}
a_{0}(\underline{v}, \underline{\hat{v}})-b_{0}(p, \underline{\hat{v}})=b_{f}\left(f ; p_{0}, \underline{\hat{v}}\right)+G_{1}(f ; \underline{\hat{v}})-a_{f}\left(f ; \underline{v}_{0}, \underline{\hat{v}}\right) \forall \hat{v} \in \mathbb{X} \\
b_{0}(\hat{p}, \underline{v})+b_{f}\left(f ; \hat{p}, \underline{v}_{0}\right)=0 \forall \hat{p} \in \mathbb{L}^{2}(\Omega), \\
\inf _{f}=\frac{\alpha}{2}\|f\|_{\mathbb{H}_{0}^{1}\left(x_{1}, x_{2}\right)}^{2}+J(f, \underline{v}, p),
\end{array}\right.
$$

where

\footnotetext{
${ }^{1}$ In this and following sections we denote $\underline{v}_{1}=\underline{v}, p_{1}=p, f_{1}=f$ but we will keep in mind that $\underline{v}, p, f$ are the "first corrections" of $\underline{v}_{0}, p_{0}, f_{0}$ on the unperturbed domain.
} 

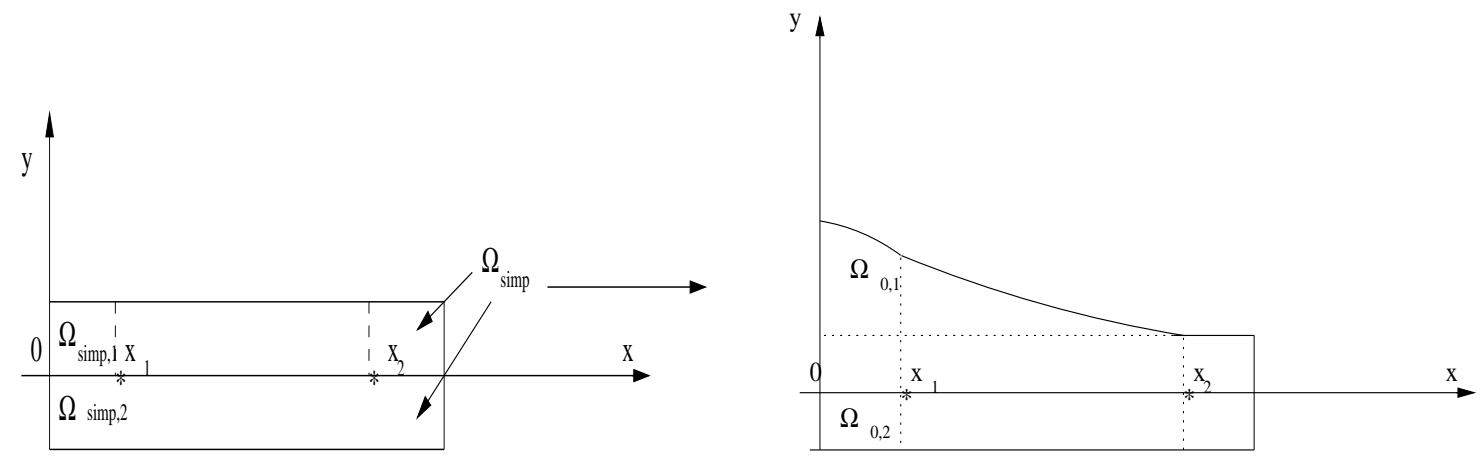

Figure 6: Subdomain $\Omega_{\text {simp }} \rightarrow \Omega_{0}$.

$$
\begin{aligned}
& a_{0}(\underline{v}, \underline{\hat{v}})=\int_{\Omega_{0}} \nu\left(\frac{\partial \underline{v}}{\partial x} \cdot \frac{\partial \underline{\hat{v}}}{\partial x}+\frac{\partial \underline{v}}{\partial y} \cdot \frac{\partial \underline{\hat{v}}}{\partial y}\right) d x d y . \\
& b_{0}(p, \underline{\hat{v}})=\int_{\Omega_{0}} p \nabla \cdot \underline{\hat{v}} d x d y, \\
& b_{f}\left(f, p_{0}, \underline{\hat{v}}\right)=\int_{\Omega_{0,1}} p_{0} \mathcal{D}_{f}(f, \underline{\hat{v}}) d x d y+\int_{\Omega_{0,1}} \frac{f}{f_{0}} p_{0} \nabla \cdot \underline{\hat{v}} d x d y, \\
& \mathcal{D}_{f}(f, \underline{\hat{v}})=-\left[y\left(\frac{f_{x} f_{0}-f_{0, x} f}{f_{0}^{2}}\right) \frac{\partial \hat{u}}{\partial y}+\frac{f}{f_{0}} \frac{\partial \hat{v}}{\partial y}\right], \\
& \mathcal{D}_{f}\left(f, v_{0}\right) \equiv \mathcal{D}_{f} f, \\
& G_{1}(f ; \underline{\hat{v}})=\int_{\Omega_{0,1}} \frac{f}{f_{0}} \underline{F} \cdot \underline{\hat{v}} d x d y, \\
& a_{f}\left(f ; \underline{v}_{0}, \underline{\hat{v}}\right)=\int_{\Omega_{0,1}} \frac{f \nu}{f_{0}} \nabla \underline{v}_{0} \cdot \nabla \underline{\hat{v}} d x d y-\int_{\Omega_{0,1}} \nu y \frac{\left(f_{x} f_{0}-f_{0, x} f\right)}{f_{0}^{2}}\left(\frac{\partial \underline{v}_{0}}{\partial y} \cdot \frac{\partial \underline{\hat{v}}}{\partial x}+\frac{\underline{v}_{0}}{\partial x} \cdot \frac{\partial \underline{\hat{v}}}{\partial y}\right) d x d y+ \\
& -\int_{\Omega_{0,1}} \frac{2 f \nu}{f_{0}} \frac{\partial \underline{v}_{0}}{\partial y} \cdot \frac{\partial \underline{\hat{v}}}{\partial y} d x d y . \\
& J(f, \underline{v}, p)=\gamma_{1} J_{1}(f, \underline{v})+\gamma_{2} J_{2}(f, \underline{v}, p), \\
& J_{1}(f, \underline{v})=\frac{1}{2} \int_{\Omega_{0}} m_{w d}\left|\nabla \times \underline{v}+m_{1} \mathcal{R}_{f} f-\mathcal{R}_{o b s, 1}\right|^{2} d x d y, \\
& \mathcal{R}_{f} f \equiv \mathcal{R}_{f}\left(f, \underline{v}_{0}\right)=-y \frac{\left(f_{x} f_{0}-f_{0, x} f\right)}{f_{0}^{2}} \frac{\partial v_{0}}{\partial y}+\frac{f}{f_{0}} \frac{\partial u_{0}}{\partial y} . \\
& \nabla \times \underline{v}=\frac{\partial v}{\partial x}-\frac{\partial u}{\partial y}, \nabla \cdot \underline{v}=\frac{\partial u}{\partial x}+\frac{\partial v}{\partial y}
\end{aligned}
$$

and $J_{2}(f, \underline{v}, p)$ are given by corresponding expressions. (For example in the case of the EXAMPLES 1-4 it is enough to replace " $\frac{f_{0}}{\beta}$ " replaced by one). The problem (28) can be written in operator form as well. To derive this form we introduce the following real spaces:

$$
\mathbb{X} \subseteq\left(\mathbb{L}^{2}(\Omega)\right)^{2} \equiv\left(\mathbb{L}^{2}(\Omega)\right)^{2 *} \subseteq \mathbb{X}^{*}, \mathbb{H}^{p} \subseteq \mathbb{L}^{2}(\Omega) \equiv \mathbb{L}^{2}(\Omega)^{*} \subseteq \mathbb{H}^{p *},
$$




$$
\begin{gathered}
\mathbb{H}_{f} \subseteq \mathbb{L}^{2}\left(x_{1}, x_{2}\right) \equiv\left(\mathbb{L}^{2}\left(x_{1}, x_{2}\right)\right)^{*} \subseteq \mathbb{H}_{f}^{*}, \\
\mathbb{W} \equiv \mathbb{X} \times \mathbb{H}^{p} \subseteq \mathbb{H}_{0} \equiv\left(\mathbb{L}^{2}(\Omega)\right)^{2} \times \mathbb{L}^{2}(\Omega) \equiv \mathbb{H}_{0}^{*} \subseteq \mathbb{W}^{*},
\end{gathered}
$$

where $\mathbb{X}$ is the Hilbert space, introduced early, (in some cases we will assume that $\mathbb{X} \equiv\left(\mathbb{H}^{2}(\Omega)\right)^{2}$ or a subset of it; $\mathbb{H}^{p}$ is a suitable space for $p$, for example $\mathbb{H}^{p} \equiv \mathbb{H}_{0}^{p}(\Omega)=\left\{p: p \in \mathbb{L}^{2}(\Omega), \int_{\Omega} p d \Omega=0\right\}$ or $\mathbb{H}^{p}=\left\{p: p \in \mathbb{H}^{1}(\Omega), p=\right.$ 0 on $\left.\Gamma_{\text {out }}\right\}$ and $\mathbb{H}^{p} \neq \mathbb{H}^{p *}$ in this case; $\mathbb{H}_{f}$ is the space for $f(x)$, it could be $\mathbb{H}_{0}^{1}\left(x_{1}, x_{2}\right) \equiv \dot{\mathbb{W}}_{2}^{1}\left(x_{1}, x_{2}\right)$, or $\mathbb{H}_{f}=\left\{f: f \in \mathbb{H}^{1}\left(x_{1}, x_{2}\right), f\left(x_{1}\right)=0\right\}$ or $\mathbb{H}_{f}=\left\{f: f \in\left(\mathbb{H}^{2} \cap \mathbb{H}_{0}^{1}\right)\left(x_{1}, x_{2}\right)\right\}$ or some other spaces (including finite dimensional spaces).

Let us consider $(28)$ in the following form: find $\underline{\Phi} \equiv(\underline{v}, p)^{T} \in\left(\mathbb{X} \times \mathbb{H}^{p}=\mathbb{W}\right)$, $f \in \mathbb{H}^{f}$, s.t

$$
\left\{\begin{array}{l}
\mathcal{L}(\underline{\Phi}, \hat{\Phi})=B(f, \hat{\Phi}) \forall \hat{\underline{\Phi}} \in \mathbb{W} \\
\inf _{f \in \mathbb{H}_{f}}=\frac{\alpha}{2}\|f\|_{\mathbb{H}^{1}}^{2}+J(f, \underline{\Phi})
\end{array}\right.
$$

where

$$
\begin{gathered}
\mathcal{L}(\underline{\Phi}, \underline{\hat{\Phi}}) \equiv a_{0}(\underline{v}, \underline{\hat{v}})-b_{0}(p, \underline{\hat{v}})+b_{0}(\hat{p}, \underline{v}), \\
B(f, \underline{\hat{\Phi}}) \equiv b_{f}\left(f, p_{0}, \underline{\hat{v}}\right)+G_{1}(f, \underline{\hat{v}})-a_{f}\left(f, \underline{v}_{0}, \underline{\hat{v}}\right)-b_{f}\left(f, \hat{p}, \underline{v}_{0}\right) \\
\underline{\hat{\Phi}}=(\underline{\hat{v}}, \hat{p}) \in \mathbb{W} .
\end{gathered}
$$

Assume $\underline{\Phi}$ to be a solution of (29). Then

$$
\alpha(f, \delta f)_{\mathbb{H}_{f}}+\left\langle J_{\Phi}^{\prime}(f, \underline{\Phi}), \delta \underline{\Phi}\right\rangle+\left\langle J_{f}^{\prime}(f, \underline{\Phi}), \delta f\right\rangle=0,
$$

where $\delta f$ is the independent variation (i.e. any function from $\mathbb{H}_{f}$ ), $\delta \underline{\Phi}$ is the dependent variation and satisfies the following equation:

$$
\mathcal{L}(\delta \underline{\Phi}, \underline{\hat{\Phi}})=B(\delta f, \underline{\hat{\Phi}}) \forall \underline{\hat{\Phi}} \in \mathbb{W}
$$

for any $\delta f \in \mathbb{H}_{f}$, and $J_{\Phi}^{\prime}=\frac{\partial J}{\partial \Phi}, J_{f}^{\prime}=\frac{\partial J}{\partial f}$ are partial derivatives of $J$, while $\langle Q, \underline{\Phi}\rangle$ is the duality between $\mathbb{W}$ and $\mathbb{W}^{*},\langle g, f\rangle$ is the duality between $\mathbb{H}_{f}$ and $\mathbb{H}_{f *}$. So, we have the system of variational equations ("optimality conditions"):

$$
\left\{\begin{array}{l}
\mathcal{L}(\underline{\Phi}, \hat{\underline{\Phi}})=B(f, \underline{\hat{\Phi}}) \forall \underline{\hat{\Phi}} \in \mathbb{W} \\
\alpha(f, \hat{f})_{\mathbb{H}^{f}}+\left\langle J_{\Phi}^{\prime}(f, \underline{\Phi}), \underline{\Phi}_{f}\right\rangle+\left\langle J_{f}^{\prime}(f, \underline{\Phi}), \hat{f}\right\rangle=0, \forall \hat{f} \in \mathbb{H}_{f}
\end{array}\right.
$$

where $\underline{\Phi}_{f} \equiv\left(\underline{v}_{f}, p_{f}\right) \equiv \underline{\Phi}_{f}(\hat{f})$ is determined by

$$
\mathcal{L}\left(\underline{\Phi}_{f}, \underline{\hat{\Phi}}\right)=B(\hat{f}, \underline{\hat{\Phi}}) \forall \underline{\hat{\Phi}} \in \mathbb{W}
$$

for chosen $\hat{f} \in \mathbb{H}_{f}$. The element $\underline{\Phi}_{f}$ can be eliminated in (32) by introducing the adjoint problem: find $\underline{Q} \equiv(\underline{q}, \sigma)^{T} \in \mathbb{W}$ s.t.

$$
\mathcal{L}^{*}(\underline{Q}, \underline{\hat{W}}) \equiv \mathcal{L}(\underline{\hat{W}}, \underline{Q})=\left\langle J_{\Phi}^{\prime}(f, \underline{\Phi}), \underline{\hat{W}}\right\rangle \forall \underline{\hat{W}} \in \mathbb{W} .
$$

Since $\underline{\Phi}_{f} \in \mathbb{W}$ then we can choose $\underline{\hat{W}}=\Phi_{f}$ in (34). Then

$$
\left.\left\langle J_{\Phi}^{\prime}(f, \underline{\Phi}), \underline{\Phi}_{f}\right)\right\rangle=\mathcal{L}\left(\underline{\Phi}_{f}, \underline{Q}\right)=B(\hat{f}, \underline{Q})
$$


and the system of variational equations reads now as follows:

$$
\left\{\begin{array}{l}
\mathcal{L}(\underline{\Phi}, \underline{\hat{\Phi}})=B(f, \hat{\hat{\Phi}}) \forall \hat{\Phi} \in \mathbb{W} \\
\mathcal{L}^{*}(\underline{Q}, \underline{\hat{W}})=\left\langle J_{\Phi}^{\prime}(f, \underline{\Phi}), \underline{\hat{W}}\right\rangle \forall \underline{\hat{W}} \in \mathbb{W}, \\
\alpha(f, \hat{f})_{\mathbb{H}_{f}}+B(\hat{f}, \underline{Q})+\left\langle J_{f}^{\prime}(f, \underline{\Phi}), \hat{f}\right\rangle=0, \forall \hat{f} \in \mathbb{H}_{f},
\end{array}\right.
$$

where the first equation is the state equation. To rewrite (36) in operator form let us define operators and elements associated with forms $\mathcal{L}(\underline{\Phi}, \underline{\hat{\Phi}}), \ldots, B(f, \underline{\Phi})$. See [12], [11], [1].

$$
\begin{gathered}
\mathcal{L}(\underline{\Phi}, \underline{\hat{\Phi}}) \equiv(L \underline{\Phi}, \underline{\hat{\Phi}})_{\mathbb{H}_{0}}, L: W \rightarrow W^{*} \forall \underline{\Phi}, \underline{\hat{\Phi}} \in \mathbb{W}, \\
\mathcal{L}^{*}(\underline{Q}, \underline{\hat{W}})=\mathcal{L}(\underline{\hat{W}}, \underline{Q})=(L \underline{\hat{W}}, \underline{Q})_{\mathbb{H}_{0}}=\left(\underline{\hat{W}}, L^{*} \underline{Q}\right)_{\mathbb{H}_{0}}, L^{*}: W \rightarrow W^{*} \forall \underline{Q}, \underline{\hat{W}} \in \mathbb{W}, \\
B(f, \underline{\Phi})=(B f, \underline{\Phi})_{\mathbb{H}_{0}} \forall f, \underline{\Phi}, B: \mathbb{H}_{f} \rightarrow \mathbb{W}^{*}, \\
\left\langle J_{\Phi}^{\prime}(f, \underline{\Phi}), \underline{\hat{W}}\right\rangle \equiv\left(\Lambda_{w} J_{\Phi}(f, \underline{\Phi}), \underline{\hat{W}}\right)_{\mathbb{H}_{0}}, \Lambda_{w}: \mathbb{W}^{*} \rightarrow \mathbb{W}^{*}, \\
\left\langle J_{f}^{\prime}(f, \underline{\Phi}), \hat{f}\right\rangle \equiv\left(\Lambda_{f} J_{f}(f, \underline{\Phi}) \hat{f}\right)_{\mathbb{L}^{2}\left(x_{1}, x_{2}\right)}, \Lambda_{f}:\left(\dot{\mathbb{W}}_{2}^{1}\right)^{*} \rightarrow\left(\dot{\mathbb{W}}_{2}^{1}\right)^{*} .
\end{gathered}
$$

Now the system (37) can be written as follows:

$$
\left\{\begin{array}{l}
L \underline{\Phi}=B f \quad\left(\text { in } \mathbb{W}^{*}\right) \\
L^{*} \underline{Q}=\Lambda_{W} J_{\Phi}(f, \underline{\Phi}) \quad\left(\text { in } \mathbb{W}^{*}\right), \\
\alpha \underline{\Lambda_{c}} f+B^{*} \underline{Q}+\Lambda_{f} J_{f}(f, \underline{\Phi})=0 \quad\left(\text { in }\left(\dot{\mathbb{W}}_{2}^{1}\right)^{*}\right)
\end{array}\right.
$$

where $\Lambda_{c}$ is the extension to $\mathbb{H}_{f}$ of the operator $\Lambda_{c, 0}$ :

$$
\Lambda_{c, 0} f \equiv-f_{x x}+f, \quad \mathcal{D}\left(\Lambda_{c, 0}\right)=\mathbb{H}^{2} \cap H_{f}
$$

REMARK. The operator form system (37) for the cost functional $J=\| C \underline{\Phi}-$ $\underline{\Psi} \|_{\mathbb{H}_{o b}}^{2}$ with operator $C: \mathbb{W} \rightarrow \mathbb{H}_{o b}$ and a given $\underline{\Psi} \in \mathbb{H}_{o b}$ is analyzed in [1]. In this case $J_{f}^{\prime}=0$ and $\Lambda_{w} J_{\Phi}^{\prime}(f, \underline{\Phi})=C^{*}(C \underline{\Phi}-\underline{\Psi})$.

\section{Uniqueness and existence results}

We analyze the particular cases where the cost functional $J$ is given by EXAMPLES 1-4 of Section 4.

7.1. Consider $J_{2}$ as in Examples 1. In this case

$$
\begin{gathered}
J(f, \underline{\Phi})=J(f, \underline{v}, p)=\frac{\gamma_{1}}{2} \int_{\Omega_{0}} m_{w d}\left|\nabla \times \underline{v}+m_{1} \mathcal{R}_{f} f-\mathcal{R}_{\text {obs }, 1}\right|^{2} d \Omega+ \\
+\gamma_{2} \int_{\Gamma_{\text {out }}}\left|p-p_{\text {out }}\right|^{2}+\left|\underline{v}-\underline{v}_{\text {out }}\right|^{2} d \Gamma
\end{gathered}
$$

To study the problems in this case we assume that $\Omega_{w d}=\Omega_{0}$ ad we put:

$$
\begin{gathered}
\mathbb{X} \equiv\left\{\underline{v}: \underline{v} \in\left(\mathbb{H}^{2}(\Omega)\right)^{2}, \underline{v}=0 \text { on } \Gamma_{i n} \cup \Gamma_{w_{1}} \cup \Gamma_{w_{3}}\right\}, \\
\mathbb{H}^{p} \equiv \mathbb{H}^{1}\left(\Omega_{0}\right), \mathbb{H}_{f} \equiv \mathbb{H}^{2}\left(x_{1}, x_{2}\right) \cap \mathbb{H}_{0}^{1}\left(x_{1}, x_{2}\right) .
\end{gathered}
$$


The derivatives $J_{\Phi}^{\prime}(f, \underline{\Phi})$ and $J_{f}^{\prime}(f, \underline{\Phi})$ are given here by the following

$$
\begin{gathered}
\left\langle J_{\Phi}^{\prime}(f, \underline{\Phi}), \underline{\hat{\Phi}}\right\rangle=\gamma_{1} \int_{\Omega_{0}} m_{w d}\left(\nabla \times \underline{v}+m_{1} \mathcal{R}_{f} f-\mathcal{R}_{o b s, 1}\right) \nabla \times \underline{\hat{v}} d \Omega+ \\
+\gamma_{2} \int_{\Gamma_{\text {out }}}\left(p-p_{\text {out }}\right) \hat{p} d \Gamma+\gamma_{2} \int_{\Gamma_{\text {out }}}\left(\underline{v}-\underline{v}_{\text {out }}\right) \cdot \underline{\hat{v}} d \Gamma,(\text { with } \underline{\hat{\Phi}}=(\underline{\hat{v}}, \hat{p})), \\
\left\langle J_{f}^{\prime}(f, \underline{\Phi}), \hat{f}\right\rangle=\gamma_{1} \int_{\Omega_{0}} m_{w d}\left(\nabla \times \underline{v}+m_{1} \mathcal{R}_{f} f-\mathcal{R}_{\text {obs }, 1}\right) \mathcal{R}_{f} \hat{f} d \Omega,
\end{gathered}
$$

The system of variational equations (32) reads: find $\underline{v} \in \mathbb{X}, p \in \mathbb{H}^{p}$

$$
\left\{\begin{array}{l}
a_{0}(\underline{v}, \underline{\hat{v}})=b_{0}(p, \underline{\hat{v}})+F(f, \underline{\hat{v}}) \forall \underline{\hat{v}} \in \mathbb{X}, \\
b_{0}(\underline{\hat{p}}, \underline{v})+b_{f}\left(f ; \hat{\hat{p}}, \underline{v}_{0}\right)=0 \forall \hat{p} \in \mathbb{H}^{p}(\Omega), \\
\alpha(\hat{f},)_{\mathbb{H}_{f}}=\gamma_{1} \int_{\Omega_{0}} m_{w d}\left(\nabla \times \underline{v}+m_{1} \mathcal{R}_{f} f-\mathcal{R}_{o b s, 1}\right) \cdot\left(\nabla \times \underline{v}_{f}+m_{1} \mathcal{R}_{f} \hat{f}\right) d \Omega+ \\
+\gamma_{2} \int_{\Gamma_{\text {out }}}\left(\left(p-p_{\text {out }}\right) p_{f}+\left(\underline{v}-\underline{v}_{\text {out }}\right) \cdot \underline{v}_{f} d \Gamma \forall \hat{f} \in \mathbb{H}_{f},\right.
\end{array}\right.
$$

where

$$
F(f, \underline{\hat{v}}) \equiv b_{f}\left(f, p_{0}, \underline{\hat{v}}\right)+G_{1}(f, \underline{\hat{v}})-a_{f}\left(f, \underline{v}_{0}, \underline{\hat{v}}\right),
$$

and $\underline{v}_{f}=\underline{v}_{f}(\hat{f}), p_{f}=p_{f}(\hat{f})$ denote the solution of the system given of the first and second equations in (39) corresponding to (any) given function $f \equiv \hat{f}$. The system (37) is: find $\underline{v} \in \mathbb{X}, p \in \mathbb{H}^{p}$

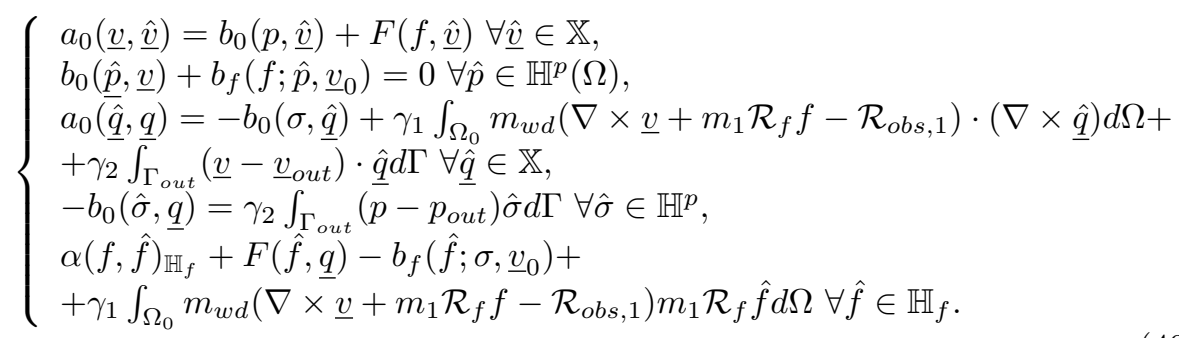

In the sequel we assume that the generalized Stokes problem has a unique solution for given $\underline{v}_{0}, p_{0}$ ( the solution in the unperturbed domain $\Omega_{0}$ ) and for each $f \in \mathbb{H}_{f}$. (See $[8]$ ).

Consider now the problem (40) for $\alpha>0$.

PROPOSITION I. For any $\alpha>0$ the problem (40) has an unique solution for each given $\underline{\mathcal{R}}_{o b s, 1}$.

Proof. Following from ([1]). After inverting $L, L^{*}$ in the first and second equations from (37) and making substitutions of expressions for $\underline{\Phi}, Q$ into the third equation we obtain the following weak statement of the problem for $f$ :

$$
\alpha(f, \hat{f})_{\mathbb{H}_{f}}+(A f, A \hat{f})_{\mathbb{L}^{2}\left(x_{1}, x_{2}\right)}=(G, A \hat{f})_{\mathbb{L}^{2}\left(x_{1}, x_{2}\right)}, \forall \hat{f} \in \mathbb{H}_{f}
$$

where $A$ is a linear operator, which depends on previous operators from variational equations, while the element $G$ will depend on the data. We see, that if $\alpha>0$ then the problem (41) has solution and $f \in \mathbb{H}_{f}:\|f\|_{\mathbb{H}_{f}}^{2} \leq\|G\|^{2} /(2 \alpha)<$ $\infty$. Using this function $f \in \mathbb{H}_{f}$ we can construct $\underline{v}, p, q, \sigma$, which jointly with $f$ is a unique solution of (40). Note that if the generalized Stokes problem is 
correctly solvable, then the problem (40) is correctly solvable too.

Consider the problem (40) as $\alpha=0$.

PROPOSITION II. Assume that: i) $\alpha=0$, ii) $\left(\frac{\partial v_{0}}{\partial y}\right)^{2}+\left(\frac{\partial u_{0}}{\partial y}\right)^{2}>0$ at $y=0, x \in\left(x_{1}, x_{2}\right)$ iii) the problem (40) has a solution. Then this solution is unique in the class $\left(\mathbb{H}^{2}(\Omega)\right)^{2} \times \mathbb{H}^{1}(\Omega)$.

Proof. Let $\underline{v}_{1}, \underline{v}_{2}, \ldots, f_{1}, f_{2}$ be two solutions of (40). Then for $\underline{v}=\underline{v}_{1}-$ $\underline{v}_{2}, \ldots, f=f_{1}-f_{2}$ from (39) we obtain:

$$
\left\{\begin{array}{l}
a_{0}(\underline{v}, \underline{\hat{v}})=b_{0}(p, \underline{\hat{v}})+F(f, \hat{\hat{v}}) \forall \underline{\hat{v}} \in \mathbb{X} \\
b_{0}(\underline{\hat{p}}, \underline{v})+b_{f}\left(f ; \hat{p}, \underline{v}_{0}\right)=0 \forall \hat{p} \in \mathbb{H}^{p}(\Omega) \\
\nabla \times \underline{v}+m_{1} \mathcal{R}_{f} f=0 \in \Omega \\
p=0, \underline{v}=0 \text { on } \Gamma_{\text {out }}
\end{array}\right.
$$

Consider the second and third equation from (42) in $\Omega_{2,0}$

$$
\nabla \cdot \underline{v}=0, \nabla \times \underline{v}=0 \text { in } \Omega_{2,0} .
$$

Then: $\Delta \underline{v}=0$ in $\Omega_{2,0}$. Considering $\underline{\hat{v}}$ with $\operatorname{supp}(\underline{\hat{v}}) \subseteq \Omega_{2,0}$ from the first equation of (42) we conclude $\nabla p=0, p=$ const in $\Omega_{2,0}$ and $-p \cdot \underline{n}+\nu \frac{\partial v}{\partial \underline{n}}=0$ on $\Gamma_{\text {out }}$. Since $p=0$ on $\Gamma_{\text {out }}$ then $p=0$ in $\Omega_{2,0}$ and $\nu \frac{\partial v}{\partial n}$ on $\Gamma_{\text {out }}$ too. So, we have the problem to $\underline{v}$ :

$$
\Delta \underline{v}=0 \text { in } \Omega_{2,0}, \underline{v}=\nu \frac{\partial \underline{v}}{\partial \underline{n}}=0 \text { on } \Gamma_{\text {out }} .
$$

This problem has only the trivial solution $\underline{v} \equiv 0$ in $\Omega_{2,0}$. Since $\underline{v} \in\left(\mathbb{H}^{2}(\Omega)\right)^{2}$ then

$$
\underline{v}=\frac{\partial \underline{v}}{\partial \underline{n}}=0 \text { on } \Gamma_{0} \equiv\left\{(x, y): y=0, x_{1}<x<x_{2}\right\} .
$$

Consider now the second and third equations from (42) in $\Omega_{1,0}$ :

$$
\left\{\begin{array}{l}
\nabla \cdot \underline{v}-\left[y\left(\frac{f_{x} f_{0}-f_{0, x} f}{f_{0}^{2}}\right) \frac{\partial u_{0}}{\partial y}+\frac{f}{f_{0}} \frac{\partial v_{0}}{\partial y}\right]=0, \text { in } \Omega_{1,0} \\
\nabla \times \underline{v}-\left[y\left(\frac{f_{x} f_{0}-f_{0, x} f}{f_{0}^{2}}\right) \frac{\partial v_{0}}{\partial y}-\frac{f}{f_{0}} \frac{\partial u_{0}}{\partial y}\right]=0, \text { in } \Omega_{1,0} .
\end{array}\right.
$$

On $\Gamma_{0}$ we have:

$$
\begin{gathered}
\nabla \cdot \underline{v}-\frac{f}{f_{0}} \frac{\partial v_{0}}{\partial y}=0, \nabla \times \underline{v}+\frac{f}{f_{0}} \frac{\partial u_{0}}{\partial y}=0, \\
|f(x)|=f_{0} \frac{\left[(\nabla \cdot \underline{v})^{2}+(\nabla \times \underline{v})^{2}\right]^{1 / 2}}{\left[\left(\frac{\partial v_{0}}{\partial y}\right)^{2}+\left(\frac{\partial u_{0}}{\partial y}\right)^{2}\right]^{1 / 2}} \text { on } \Gamma_{0} .
\end{gathered}
$$

Since $\underline{v}=\frac{\partial \underline{v}}{\partial \underline{n}}=\frac{\partial \underline{v}}{\partial y}=0$ on $\Gamma_{0}$, then

$$
\left.\nabla \cdot \underline{v}\right|_{y=0}=\frac{\partial u}{\partial y}+\left.\frac{\partial v}{\partial y}\right|_{y=0}=0, \nabla \times\left.\underline{v}\right|_{y=0}=\frac{\partial v}{\partial y}-\left.\frac{\partial u}{\partial y}\right|_{y=0}=0, x \in\left(x_{1}, x_{2}\right) .
$$

i.e. $f(x)=0$. Therefore, $\underline{v} \equiv 0, p=0$ too. 
7.2. The proves of uniqueness results for $J_{2}$ from EXAMPLES 2-4 are similar, therefore we consider here only $J_{2}$ of the form from EXAMPLE 3.

Assume that:

$$
\begin{gathered}
\Omega_{w d}=\Omega_{0,1}=\Omega_{0}, \gamma_{2}=0, \int_{\Omega_{0}} p d \Omega=0 \\
J=\frac{\gamma_{1}}{2} \int_{\Omega_{0}}\left|\nabla \times \underline{v}+\mathcal{R}_{f} f-\mathcal{R}_{o b s, 1}\right|^{2} d \Omega+\frac{\gamma_{2}}{2} \int_{\Gamma_{3}}\left|\frac{\partial \underline{v}}{\partial n}-g_{1, o b s}\right|^{2} d \Gamma \\
\mathbb{X}=\left\{\underline{v}: \underline{v} \in\left(\mathbb{H}^{2}(\Omega)\right)^{2}, \underline{v}=0 \text { on } \Gamma_{i n} \cup \Gamma_{w_{1}} \cup \Gamma_{w_{3}}\right\}, \\
\mathbb{H}^{p}=\left\{p: p \in \mathbb{H}^{1}\left(\Omega_{0}\right), \int_{\Omega_{0}} p d \Omega=0\right\}, \mathbb{H}_{f}=\mathbb{H}_{0}^{1}\left(x_{1}, x_{2}\right)
\end{gathered}
$$

The system of variational equations reads here as follows:

$$
\left\{\begin{array}{l}
a_{0}(\underline{v}, \underline{\hat{v}})=b_{0}(p, \underline{\hat{v}})+F(f, \underline{\hat{v}}) \forall \underline{\hat{v}} \in \mathbb{X}, \\
b_{0}(\hat{\hat{p}}, \underline{v})+b_{f}\left(f ; \hat{\hat{p}}, \underline{v}_{0}\right)=0 \forall \hat{p} \in \mathbb{H}^{p}(\Omega), \\
\alpha(f,)_{\mathbb{H}_{f}}+\gamma_{1} \int_{\Omega_{0}}\left(\nabla \times \underline{v}+\mathcal{R}_{f} f-\mathcal{R}_{o b s, 1}\right) \cdot\left(\nabla \times \underline{v}_{f}+\mathcal{R}_{f} \hat{f}\right) d \Omega+ \\
+\gamma_{2} \int_{\Gamma_{w_{3}}}\left(\frac{\partial \underline{v}}{\partial \underline{\underline{n}}}-g_{o b s}\right) \cdot \frac{\partial \underline{v}_{f}}{\partial \underline{\underline{n}}} d \Gamma=0 \forall \hat{f} \in \mathbb{H}_{f},
\end{array}\right.
$$

The system (37)reads in this case as follows:

$$
\left\{\begin{array}{l}
a_{0}(\underline{v}, \underline{\hat{v}})=b_{0}(p, \underline{\hat{v}})+F(f, \underline{\hat{v}}) \forall \underline{\hat{v}} \in \mathbb{X}, \\
b_{0}(\underline{\hat{p}}, \underline{v})+b_{f}\left(f ; \hat{p}, \underline{v}_{0}\right)=0 \forall \hat{p} \in \mathbb{H}^{p}(\Omega), \\
a_{0}(\underline{\hat{\hat{q}}}, \underline{q})=-b_{0}(\sigma, \underline{\hat{q}})+\gamma_{1} \int_{\Omega_{0}}\left(\nabla \times \underline{v}+\mathcal{R}_{f} f-\mathcal{R}_{o b s, 1}\right) \cdot(\nabla \times \underline{\hat{q}}) d \Omega+ \\
+\gamma_{2} \int_{\Gamma_{w_{3}}}\left(\frac{\partial \underline{v}}{\partial \underline{\underline{n}}}-g_{1, o b s}\right) \cdot \frac{\partial \hat{\underline{q}}}{\partial \underline{\underline{n}}} d \Gamma \forall \underline{\hat{q}} \in \mathbb{X}, \\
-b_{0}(\hat{\sigma}, \underline{q})=0 \forall \hat{\sigma} \in \mathbb{H}^{p}, \\
\alpha(f, \hat{f})_{\mathbb{H}_{f}}+F(\hat{f}, q)-b_{f}\left(\hat{f} ; \sigma, \underline{v}_{0}\right)+ \\
+\gamma_{1} \int_{\Omega_{0}}\left(\nabla \times \underline{v}+\mathcal{R}_{f} f-\mathcal{R}_{o b s, 1}\right) \mathcal{R}_{f} \hat{f} d \Omega \forall \hat{f} \in \mathbb{H}_{f} .
\end{array}\right.
$$

PROPOSITION III. The problem (45) for $\alpha>0$ has a solution and this solution is unique.

(The proof repeats the considerations of the proof of PROPOSITION I.)

PROPOSITION IV. If $i) \alpha=0$, ii) $\Omega_{w d}=\Omega_{0}=\Omega_{0,1}$, iii) $\left(\frac{\partial v_{0}}{\partial y}\right)^{2}+\left(\frac{\partial u_{0}}{\partial y}\right)^{2}>$ 0 on $\Gamma_{w_{3}}$ as $x \in\left(x_{1}, x_{2}\right)$, iv) the problem (45) has a solution. Then this solution is unique. ProOF. For the differences $\underline{v}=\underline{v}_{1}-\underline{v}_{2}, \ldots, f=f_{1}-f_{2}$ two possible solutions $\underline{v}_{1}, \underline{v}_{2}, \cdots, f_{1}, f_{2}$ we obtain the following system on $\Gamma_{w_{3}}$ with $x \in\left(x_{1}, x_{2}\right)$ (i.e. at $y=0$ with $x \in\left(x_{1}, x_{2}\right)$ ):

$$
\frac{\partial v}{\partial y}-\frac{f}{f_{0}} \frac{\partial u_{0}}{\partial y}=0,-\frac{\partial u}{\partial y}+\frac{f}{f_{0}} \frac{\partial v_{0}}{\partial y}=0, \frac{\partial \underline{v}}{\partial n}=-\left(\frac{\partial u}{\partial y}, \frac{\partial v}{\partial y}\right)=0 .
$$

Hence: $f=0 \Rightarrow p=0 \Rightarrow \underline{v}=0$.

Let us once more note, that if $\gamma_{2}>0$ and we introduce into considerations the cost functional $J_{2}$, then we overdeterminate the problem (16) for $\alpha=0$ and the initial problem. Therefore in this case we have usually uniqueness results, however not existence results generally. But in some physical problems the above overdeterminations (and the term $\alpha\|f\|_{\mathbb{H}_{f}}^{2}$ also) are reasonable and have a physical sense, therefore in these cases we can consider the optimal 
control problems like (17) as the problems to be independent of the initial problem (where we have only $J_{1}$ ). Here, we have also existence results and can name these optimal control problems as the "optimal shape design problems". Nevertheless, it is interesting to study solvability results of above variational problems as $\alpha=\gamma_{2}=0$.

\section{$7 \quad$ Iterative Processes}

Consider some iterative processes for solving the variational equations obtained in previous sections.

8.1. Consider the problem (37). One of the simplest iterative algorithms to solve (37) consists of the following steps: if $f^{(k)}$ is known from previous steps of the iterative process, then the next approximation $f^{(k+1)}$ can be determinated by solving the following equations ([1])

$$
\left\{\begin{array}{l}
L \Phi^{(k)}=B f^{(k)} \\
L^{*} \underline{Q}^{(k)}=\Lambda_{W} J_{\Phi}\left(\underline{\Phi}^{(k)}, f^{(k)}\right) \\
\Lambda_{c} w^{(k)}=B^{*} Q^{(k)}+\Lambda_{f} J_{f}\left(\underline{\Phi}^{(k)}, f^{(k)}\right), \\
f^{(k+1)}=f^{(k)}-\tau_{k}\left(\alpha f^{(k)}+w^{(k)}\right), \quad k=0,1, \ldots
\end{array}\right.
$$

where $\left\{\tau_{k}\right\}$ parameters of the iterative process, determinated by propositions of the extremal problems theory ([24]), of the general theory of iterative processes ([14], [20], [21]), of the ill-posed problems theory ([22] and [23]). Usually to prove the convergence of (46) and to make a choice of $\left\{\tau_{k}\right\}$ we need to impose some additional restrictions to $J$ (convexity,...). In terms of the system (36) the iterative process $(46)$ reads:

$$
\left\{\begin{array}{l}
\mathcal{L}\left(\underline{\Phi}^{(k)}, \hat{\Phi}\right)=B\left(f^{(k)}, \hat{\Phi}\right) \forall \hat{\Phi} \in \mathbb{W} \\
\mathcal{L}\left(\underline{\hat{W}}, \underline{Q}^{(k)}\right)=\left\langle J_{\Phi}^{\prime}\left(\underline{\Phi}^{(k)}, f^{(k)}\right), \underline{\hat{W}}\right\rangle \forall \underline{\hat{W}} \in \mathbb{W} \\
\left(w^{(k)}, \hat{f}\right)_{\mathbb{H}_{f}}=B\left(\hat{f}, \underline{Q}^{(k)}\right)+\left\langle J_{f}^{\prime}\left(\underline{\Phi}^{(k)}, f^{(k)}\right), \hat{f}\right\rangle \forall \hat{f} \in \mathbb{H}_{f} \\
f^{(k+1)}=f^{(k)}-\tau_{k}\left(\alpha f^{(k)}+w^{(k)}\right), \quad k=0,1, \ldots
\end{array}\right.
$$

Using approaches from ([1]) other iterative processes to solve the problems formulated above can be proposed too.

8.2. Consider the problem (39) (with $\Omega_{w d} \subseteq \Omega$ for the generalization). The iterative process (47) for this problem is written as follows:

$$
\left\{\begin{array}{l}
a_{0}\left(\underline{v}^{(k)}, \hat{v}\right)=b_{0}\left(p^{(k)}, \hat{v}\right)+F\left(f^{(k)}, \hat{\hat{v}}\right) \forall \underline{\hat{v}} \in \mathbb{X}, \\
b_{0}\left(\underline{\hat{p}}^{(\underline{v}} \underline{\underline{v}}^{(k)}\right)+b_{f}\left(f^{(k)} ; \hat{p}, \underline{v}_{0}\right)=0 \forall \hat{p} \in \mathbb{H}^{p}(\Omega), \\
\left(w^{(k)}, \hat{f}\right)_{\mathbb{H}_{f}}=\gamma_{1} \int_{\Omega_{0}} m_{w d}\left(\nabla \times \underline{v}^{(k)}+m_{1} \mathcal{R}_{f} f^{(k)}-\mathcal{R}_{\text {obs, }}\right) . \\
\cdot\left(\nabla \times \underline{v}_{f}+m_{1} \mathcal{R}_{f} \hat{f}\right) d \Omega+ \\
+\gamma_{2} \int_{\Gamma_{\text {out }}}\left(\left(\left(^{(k)}-p_{\text {out }}\right) p_{f}+\left(\underline{v}^{(k)}-\underline{v}_{\text {out }}\right) \cdot \underline{v}_{f} d \Gamma \forall \hat{f} \in \mathbb{H}_{f},\right.\right. \\
f^{(k+1)}=f^{(k)}-\tau_{k}\left(\alpha f^{(k)}+w^{(k)}\right), \quad k=0,1, \ldots
\end{array}\right.
$$


or using the adjoint problem:

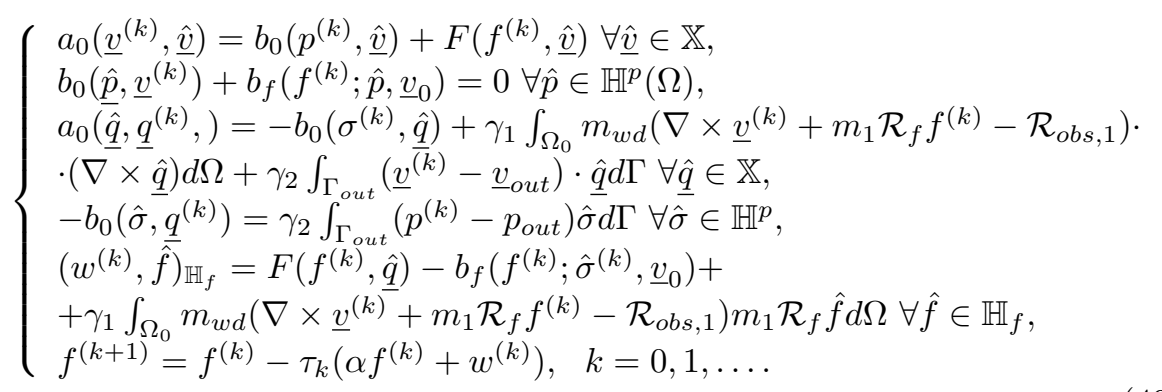

Let us assume $f,\left\{f^{(k)}\right\}, \hat{f}$ to be from a finite-dimensional subspace $\mathbb{H}_{f, N} \subset \mathbb{H}_{f}$ of dimension $N<\infty$ and with a basis $\varphi_{i}, i=1,2, \ldots, N$. Then the following statements hold true.

THEOREM. Assume that $\Omega_{w d}=\Omega,\left(\frac{\partial v_{0}}{\partial y}\right)^{2}+\left(\frac{\partial u_{0}}{\partial y}\right)^{2}>0$ at $y=0, x \in\left(x_{1}, x_{2}\right)$ and $f,\left\{f^{(k)}\right\}, \hat{f}$ are from $\mathbb{H}_{f, N} \subset \mathbb{H}_{f}$. Then the following statements are valid:

1. The problem (39) is correctly solvable for $\alpha \geq 0$ and $N<\infty$.

2. The iterative process (48) ((49)) is convergent for any $\alpha>0, N<\infty$ and for small $\tau=\tau_{k}>0, k=0,1,2, \ldots$.

3. If $\alpha$ is sufficiently small while $k=K$-sufficiently large- then $\underline{v}^{(k)}, p^{(k)}, f^{(k)}$ can be taken as an approximate solution of the problem (39).

\section{PROOF.}

1. The existence of the solution for $\alpha>0$ has been proved early. Let us consider the case $\alpha=0$. Since $f=\Sigma_{i=1}^{N} a_{i} \varphi_{i} \in \mathbb{H}_{f, N}$ then in the form (41) with $\alpha=0$ we conclude that this equation is correctly solvable (because the problem (39) can have only unique solution in $\mathbb{X} \times \mathbb{H}^{p} \times \mathbb{H}^{p}$, see PROPOSITION II). We assume the generalized Stokes problem to be correctly solvable for given $f \in \mathbb{H}_{f}$. Hence the problem (39) is correctly solvable.

2. If $\alpha>0$ the operator of the equation for $f$ (this equation in the weak form is given by (41)) is positive definite and bounded. Then according the general theory of iterative algorithm the process is given by

$$
\begin{gathered}
\left(f^{(k+1)}, \hat{f}\right)_{\mathbb{H}_{f}}=\left(f^{(k)}, \hat{f}\right)_{\mathbb{H}_{f}}-\tau\left(\alpha\left(f^{(k)}, \hat{f}\right)_{\mathbb{H}_{f}}+\left(A f^{(k)}, A \hat{f}\right)_{\mathbb{L}^{2}\left(x_{1}, x_{2}\right)}-\right. \\
-(G, A \hat{f})_{\mathbb{L}^{2}\left(x_{1}, x_{2}\right)}, k=0,1, \ldots
\end{gathered}
$$

is convergent for small $\tau>0$. Hence the process (48) ((49)) is convergent also and

$$
\left\|\underline{v}^{(k)}-\underline{v}\right\|_{\mathbb{X}}+\left\|p^{(k)}-p\right\|_{\mathbb{H}^{p}}+\left\|f-f^{(k)}\right\| \rightarrow 0, k \rightarrow \infty .
$$


3. Let $\underline{v}_{e x}, p_{e x}, f_{e x}$ be a solution of (39) as $\alpha=0$. According to he theory of ill-posed problem ([22] and [23]) we have: $\left\|f_{e x}-f\right\|_{\mathbb{H} p} \rightarrow 0$ as $\alpha \rightarrow+0$, where $(f, \underline{v}, p)$ is one solution of (39) for $\alpha>0$. Hence

$$
\left\|\underline{v}_{e x}-\underline{v}\right\|_{\mathbb{X}}+\left\|p_{e x}-p\right\|_{\mathbb{H}^{p}} \rightarrow 0, \text { as } \alpha \rightarrow+0 .
$$

From these statements and from (50) we conclude that the latter conclusion of this theorem holds true also.

Consider the simple example, which can be very useful in solving the above problems for small $N$ (the dimension of $\mathbb{H}_{f, N}$ ).

EXAMPLE. Consider the case when $N=1$ and $\mathbb{H}_{f, 1}$ is one-dimensional subspace of $\mathbb{H}_{f}$, with the unique basis function $\varphi_{1}$, the shape function:

$$
\mathbb{H}_{f, 1}=\left\{f: f=a \varphi_{1}(x), \varphi_{1} \in \mathbb{H}_{f}, a \in \mathbb{R}^{1}\right\} .
$$

Consider the problem (39) and introduce the model solution of $\underline{V}_{1}, P_{1}$ of the generalized Stokes problem with $f=\varphi_{1}$ :

$$
\left\{\begin{array}{l}
a_{0}\left(\underline{V}_{1}, \hat{\underline{\hat{v}}}\right)=b_{0}\left(P_{1}, \hat{\underline{v}}\right)+F\left(\varphi_{1}, \underline{\hat{v}}\right) \forall \underline{\hat{v}} \in \mathbb{X} \\
b_{0}\left(\underline{\hat{p}}, \underline{V}_{1}\right)+b_{f}\left(\varphi_{1} ; \hat{p}, \underline{v}_{0}\right)=0 \forall \hat{p} \in \mathbb{H}^{p}(\Omega),
\end{array}\right.
$$

It's easy to see that the exact solution of (39) is the following one:

$$
\underline{v}=a \underline{V}_{1}, p=a \underline{P}_{1}, f=a \varphi_{1},
$$

where

$a=\frac{\gamma_{1} \int_{\Omega_{0}} m_{w d} \mathcal{R}_{\text {obs }, 1}\left(\nabla \times \underline{V}_{1}+m_{1} \mathcal{R}_{f} \varphi_{1}\right) d \Omega+\gamma_{2} \int_{\Gamma_{\text {out }}}\left(P_{1} p_{\text {out }}+\underline{V}_{1} \cdot \underline{v}_{\text {out }}\right) d \Gamma}{\alpha\left\|\varphi_{0}\right\|_{\mathbb{H}_{f}}^{2}+\gamma_{1} \int_{\Omega_{0}} m_{w d}\left|\nabla \times \underline{V}_{1}+m_{1} \mathcal{R}_{f} \varphi_{1}\right|^{2} d \Omega+\gamma_{2} \int_{\Gamma_{\text {out }}}\left(P_{1}^{2}+\left|\underline{V}_{1}\right|^{2}\right) d \Gamma}$.

If $\Omega_{w d} \cap \Omega_{1}=0$ and $\gamma_{2}=0$ then

$$
a=\frac{\gamma_{1} \int_{\Omega_{0}} m_{w d} \mathcal{R}_{o b s, 1}\left(\nabla \times \underline{V}_{1}\right) d \Omega}{\alpha\left\|\varphi_{0}\right\|_{\mathbb{H}_{f}}^{2}+\gamma_{1} \int_{\Omega_{0}} m_{w d}\left|\nabla \times \underline{V}_{1}\right|^{2} d \Omega}
$$

From above expressions we see that if the function $\mathcal{R}_{o b s, 1}\left(\nabla \times \underline{V}_{1}\right)$ is smooth and is changing its sign in $\Omega_{0}$, then for some controlled domain $\Omega_{w d}$ we have $a>0$ for some others $(a<0)$, i.e the correction of $\partial \Omega_{0}$ must be made into $\mathbb{R}^{2} \backslash \Omega_{0}$ or into $\Omega_{0}$. So the optimal shape of $\partial \Omega$ depends as from vorticities $\mathcal{R}_{o b s, 1}, \nabla \times \underline{v}_{1}, \ldots$ as well as from the domain $\Omega_{w d}$ which is controlled. This example can be generalized to some other cases with small $N,(N=2,3)$ (see Fig.7) and the formulas like (52) can be useful in solving some model problems without iterative procedures.

In conclusion of this section we note that the statements of the above theorem can be proved also for the cost functional $J_{2}$ from EXAMPLES 2-4.

Assume that we calculate the approximate solution $\underline{v}^{(k)}, p^{(k), f^{(k)}}$ in the domain $\Omega_{0}$. Then the approximate solution in the domain $\Omega$ with $\Gamma_{c, \varepsilon}$ described approximately as

$$
\Gamma_{c, \varepsilon} \cong\left\{(\tilde{x}, \tilde{y}): y \cong f_{0}(x)+\varepsilon f^{(k)}(x), x \in\left(x_{1}, x_{2}\right)\right\}
$$




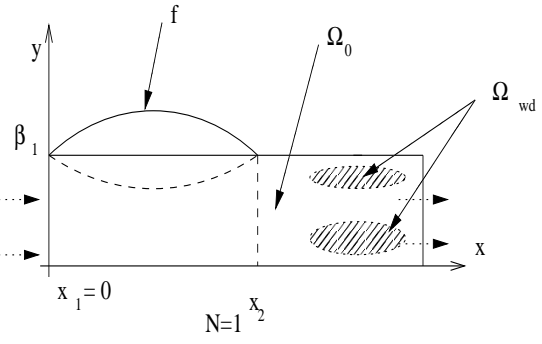

(a)

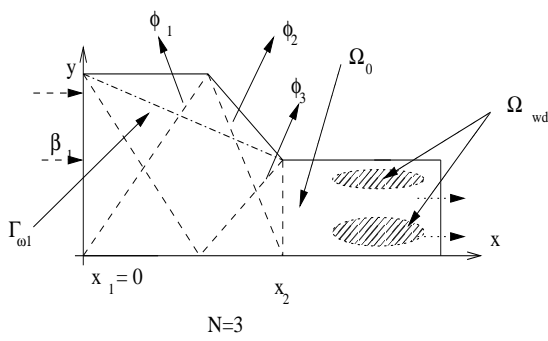

(b)

Figure 7: Domain $\Omega$ with $N$ shape functions: (a) $N=1, f=\beta_{1}+a \varphi_{0}(x)$, $\varphi_{0}=x\left(x_{2}-x\right)$; (b) $N=3, f=\beta_{1}+\Sigma_{i=1}^{3} a_{i} \varphi_{i}$.

can be obtained using the variables transformations, which are inverse to the transformations applied above. Then we have:

$$
\begin{aligned}
\underline{v}(\tilde{x}, \tilde{y}) \cong\left(\underline{v}_{0}+\varepsilon \underline{v}^{(k)}\right)\left(x, \frac{y f_{0}(x)}{f_{0}(x)+\varepsilon f^{(k)}(x)}\right) \\
p(\tilde{x}, \tilde{y}) \cong\left(p_{0}+\varepsilon p^{(k)}\right)\left(x, \frac{y f_{0}(x)}{f_{0}(x)+\varepsilon f^{(k)}(x)}\right) \\
(\tilde{x}, \tilde{y}) \in \Omega, \tilde{x}=x, \tilde{y}=\frac{y f_{0}(x)}{\left(f_{0}(x)+\varepsilon f^{(k)}(x)\right)} \\
(x, y) \in \Omega_{0}
\end{aligned}
$$

(Of course, if we know $f \cong f_{0}(x)+\varepsilon f^{(k)}(x)$, then we can solve the Stokes problem in the new domain $\Omega^{(k)} \equiv \Omega(f)$ independently.).

\section{Test Problem and Numerical Results}

To test the methods from previous sections some test problems have been proposed using simplified configuration. Numerical simulations have been carried out using Bamg [10], a Bi-dimensional Anisotropic Mesh Generator and FreeFem, a finite element Library developed at INRIA [9], the French National Institute for Research in Computer Science and Control, with the development of algorithms based on control theory and adjoint formulation for generalized Stokes problem. In this section we are going to present numerical results applying first vorticity reduction in the downfield zone of the new incoming branch of the bypass. Some remarks dealing with the studied configuration :

- Wall curvature was considered only in the zone of the incoming branch of the bypass $f_{0}=\sin (x)$, in other part we used piecewise constant function.

- Graft angle of the bypass incoming branch (which influences vorticity) was considered be zero (between the artery and the new incoming branch there isn't a relative angle).

- After obtaining the shape for the incoming branch in 25 iterations, vorticity reduction in downfield zone is about of $30 \%$, 
Velocity values $\underline{v}_{i n}$ at the inflow are chosen in such a way that the Reynolds number $R e=\frac{\bar{v} * D}{\nu}$ has order $10^{3}$. Blood kinematic viscosity $\nu=\frac{\mu}{\rho}$ is to $4 *$ $10^{-6} \mathrm{~m}^{2} \mathrm{~s}^{-1}$, blood density $\rho=1 \mathrm{~g} \mathrm{~cm}^{-3}$ and dynamic viscosity $\mu=4 *$ $10^{-2} \mathrm{~g} \mathrm{~cm}^{-1} \mathrm{~s}^{-1} ; \bar{v}$ is a mean inflow velocity related with $\underline{v}_{i n}$, while $D$ is the arterial diameter $(3.5 \mathrm{~mm})$. [18].

Figures (8)-(10) provide a preliminary account of numerical results and show how the shape of the bypass using genaralized steady Stokes equations in an optimal control problem is smoothed at the corner.
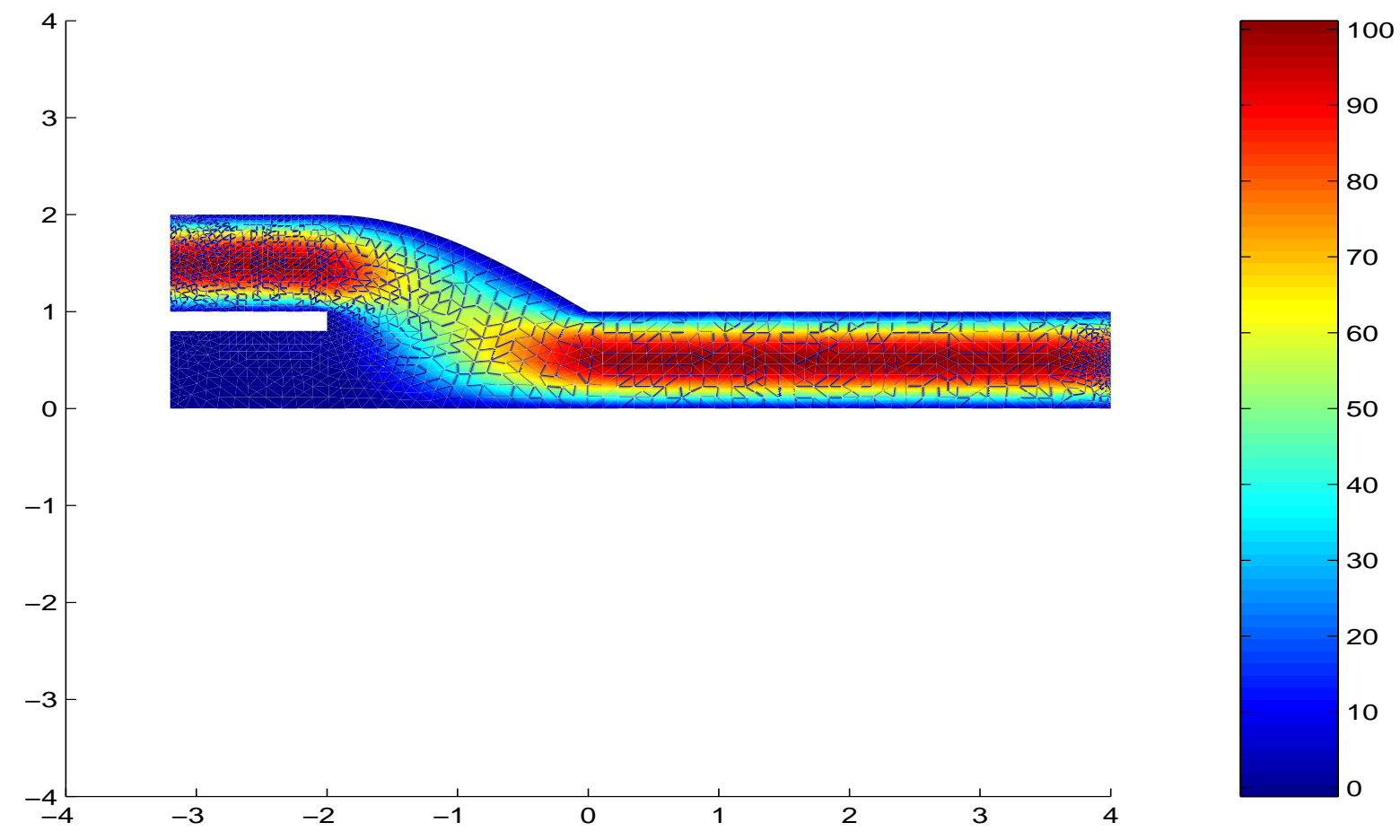

Figure 8: Idealized, 2-D bypass configuration before optimal shape design process: iso-velocity $\left[\mathrm{cms}^{-1}\right]$. 


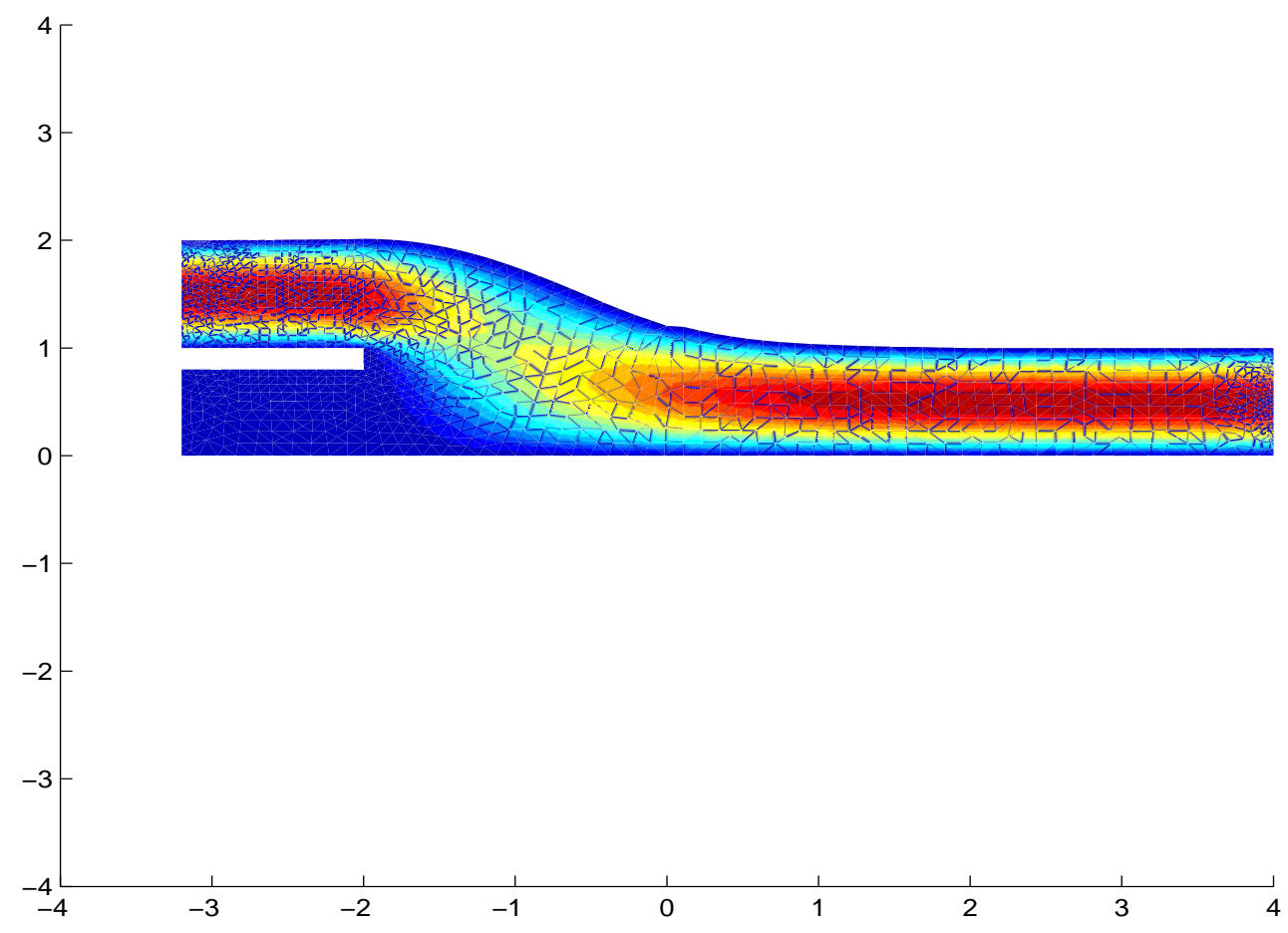

Figure 9: Bypass configuration at the end of shape optimization using first corrections: iso-velocity. 


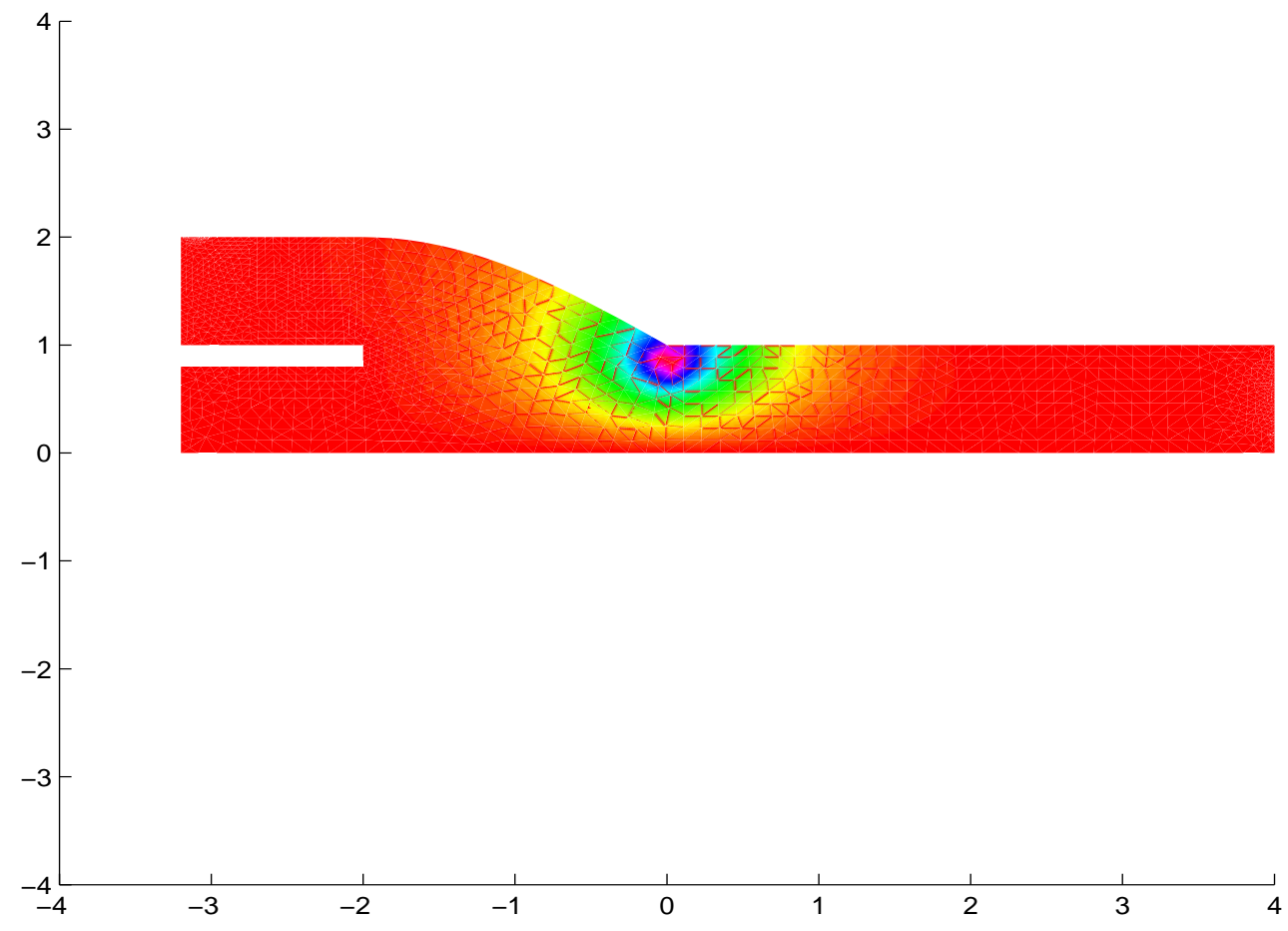

Figure 10: Adjoint solution $\underline{q}$ in Bypass configuration in the reference domain. 


\section{$9 \quad$ Future Developments}

The development of tools for geometry reconstruction from medical data (medical imaging and other non-invasive means) and their integration with numerical simulation could provide improvements in disease diagnosis procedures.

We draw attention to possibilities for using our approaches and results in sensitivity analysis of problems considered early. Indeed, it is easy to see that the problem for first corrections is really the problem for first variations in the sensitivity analysis.

Using the numerical method developed in this paper it is possible to realize the iterative process for solving initial nonlinear problem. To do this it is sufficient to put the function $f=f_{0}+\varepsilon f$ as the new $f_{0}$, to calculate new first corrections and so on.

Optimal control and shape optimization applied to fully unsteady incompressible Stokes and Navier-Stokes equations and possibly the coupled fluid-structure problem and the setting of the problem in a three-dimensional geometry will provide more realistic design indications concerning surgical prosthesis realizations. A further development will be devoted to build domain decomposition methods based on optimal control approaches and efficient schemes for reduced-basis methodology approximations which could be more efficient for use in a repetitive design environment as optimal shape design methodology requires.

\section{Acknowledgements}

Bernoulli Center of EPFL is acknowledged for the support of the authors during the special semester on the "Mathematical Modelling of the Cardiovascular System". G.Rozza acknowledges the financial support provided through the European Community's Human Potential Programme under contract HPRN-CT2002-00270 HaeMOdel. This work has also been supported in part by the Swiss National Science Foundation (Project n. 20-65110.01) and by Italian Cofin2001 MURST Project "Metodi numerici avanzati per equazioni alle derivate parziali di interesse applicativo".

\section{References}

[1] V.I. Agoshkov. Optimal Control Approaches and Adjoint Equations in the Mathematical Physics Problems. - Institute of Numerical Mathematics, Russian Academy of Sciences, Moscow, 2003.

[2] J.S. Cole, J.K. Watterson, M.J.G. O'Reilly. Numerical investigation of the haemodynamics at a patched arterial bypass anastomosis. Medical Engineering and Physics, Vol. 24, 2002, pp. 393-401.

[3] J.S. Cole, J.K. Watterson, M.J.G. O'Reilly. Is there a haemodynamic advantage associated with cuffed arterial anastomoses. Journal of Biomechanics, Vol. 35, 2002, pp. 1337-46.

[4] J.S. Cole, L.D. Wijesinghe, J.K. Watterson and D.J.A. Scott. Computational and Experimental Simulations of the Haemodynamics at Cuffed Arterial Bypass Graft Anastomoses. Proceedings of the Institution of Mechan- 
ical Engineers, Part H: Journal of Engineering in Medicine, Vol. 216, 2002, pp. 135-143.

[5] R.Courant, D. Hilbert. Methods of Mathematical Physics. Wiley, New York, 1966.

[6] Y.C. Fung. Biodynamics: Circulation. Springer and Verlag, New York, 1984.

[7] G.P. Galdi An Introduction to the Mathematical Theory of the NavierStokes Equations, Linearized Steady Problem. Springer-Verlag, New York, 1994.

[8] V.Girault, P.A. Raviart Finite Element Methods for Navier-Stokes Equations. Springer-Verlag, Berlin, 1986.

[9] F.Hecht, O.Pironneau, K.Ohtsuka. Freefem++ Manual 1.34. http://www.freefem.org, 2003.

[10] F. Hecht. BAMG: Bidimensional Anisotropic Mesh Generator. User Guide. INRIA, Rocquencourt, 1998.

[11] J.L. Lions. Optimal Control of Systems Governed by Partial Differential Equations. Springer-Verlag, 1971.

[12] J.L. Lions, E. Magenes. Non-homogeneous Boundary Value Problems and Applications. Springer-Verlag, 1972.

[13] F. Loth, S.A Jones, D.P. Giddens, H.S Bassiouny, C.K Zarins, S. Glagov. Measurement of Velocity and Wall Shear Stress inside a PTE vascular graft model under steady flow conditions. Journal of Biomechanical Engineering, Vol. 119, pp. 187-194, May 1997.

[14] G.I. Marchuk. Methods of Numerical Mathematics. Nauka, Moscow, 1989.

[15] J.A. Moore, D.A. Steinman, S. Prakash, C.R. Ethier, K.W Johnston. A numerical study of blood flow patterns in anatomically realistic and simplified end-to-side anastomoses. ASME, J.Biomechanical Engineering, Vol. 121(3), 1999, pp. 265-72.

[16] K. Perktold, M. Hofer, G. Karner, W. Trubel, H. Schima. Computer Simulation of Vascular Fluid Dynamics and Mass Transport: Optimal Design of Arterial Bypass Anastomoses. Proceedings of ECCOMAS 98, pp. 484-489, K. Papailion and others Editors, John Wiley and Sons, Ltd, 1998.

[17] A. Quarteroni, G.Rozza. Optimal Control and Shape Optimization in Aorto-Coronaric Bypass Anastomoses. $M^{3} A S$ Mathematical Models and Methods in Applied Sciences, Vol. 13, No.12 (2003), pp. 1-23.

[18] A. Quarteroni, L. Formaggia. Mathematical Modelling and Numerical Simulation of the Cardiovascular System in Modelling of Living Systems, Handbook of Numerical Analysis Series (P.G. Ciarlet e J.L. Lions Eds), Elsevier, Amsterdam, 2003. 
[19] A. Quarteroni, M. Tuveri, A. Veneziani. Computational Vascular Fluid Dynamics: Problems, Models and Methods. Computing and Visualization in Science, Vol. 2 (2000), pp. 163-197.

[20] A. Quarteroni, A. Valli. Numerical Approximation of Partial Differential Equations. Springer-Verlag, Berlin, 1994.

[21] A. Quarteroni, R. Sacco, F. Saleri. Numerical Mathematics. Springer, New York, 2000.

[22] A.N. Tikhonov, V.Ya. Arsenin. Methods for solving ill-posed problems. Nauka, Moscow, 1974.

[23] G.M. Vainikko, A.Yu. Veretennikov. Iterative Procedures in ill-posed problems. Nauka, Moscow, 1986.

[24] F.P. Vasiliev. Methods for solving the extremum problems. Nauka, Moscow, 1981. 